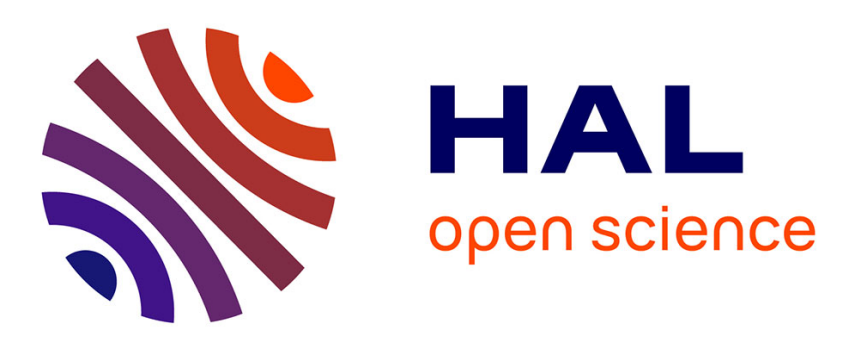

\title{
Emulating an Anechoic Environment in a Wave-Diffusive Medium through an Extended Time-Reversal Approach
}

Andrea Cozza

\section{To cite this version:}

Andrea Cozza. Emulating an Anechoic Environment in a Wave-Diffusive Medium through an Extended Time-Reversal Approach. IEEE Transactions on Antennas and Propagation, 2012, 60 (8), pp.3838-3852. 10.1109/TAP.2012.2201097 . hal-00682795v2

\section{HAL Id: hal-00682795 \\ https://hal.science/hal-00682795v2}

Submitted on 10 Jun 2015

HAL is a multi-disciplinary open access archive for the deposit and dissemination of scientific research documents, whether they are published or not. The documents may come from teaching and research institutions in France or abroad, or from public or private research centers.
L'archive ouverte pluridisciplinaire HAL, est destinée au dépôt et à la diffusion de documents scientifiques de niveau recherche, publiés ou non, émanant des établissements d'enseignement et de recherche français ou étrangers, des laboratoires publics ou privés. 


\title{
Emulating an Anechoic Environment in a Wave-Diffusive Medium through an Extended Time-Reversal Approach
}

\author{
Andrea Cozza, Member
}

\begin{abstract}
A generalized time-reversal (TR) technique for the generation of coherent wavefronts within complex media is presented in this paper. Although completely general, this method is primarily considered for testing purposes herein, where an equipment under test is submitted to a series of impinging wavefronts with varying features. Electromagnetic compatibility, antenna testing as well as telecommunications facilities where complex-wavefront schemes (e.g., multi-path configurations) are required, could benefit from the proposed approach. The main advantages and limitations of current standard TR approaches are reviewed in this respect, exposing their inadequacy for this particular context. The proposed alternative technique, named Time-Reversal Electromagnetic Chamber (TREC) is introduced and studied by means of a formal theoretical analysis, showing how a reverberation chamber (RC) supporting a diffused-field condition can be operated as a generator of deterministic pulsed wavefronts. The TREC is demonstrated to be capable of generating arbitrary wavefronts with a remarkable accuracy, allowing to revisit the $\mathrm{RC}$ as a deterministic facility: the main advantages of RCs and anechoic ones are merged, leading to a new facility capable of potentially generating in real-time pulsed wavefronts while using low input energies, without requiring neither mechanical displacements nor any special features of the sources.
\end{abstract}

Index Terms-Cavities, random media, test facilities, timedomain measurements, dyadic Green's functions, wave focusing, time reversal.

\section{INTRODUCTION}

$\mathbf{T}$ HE idea of assessing the response of an equipment under test (EUT) to external electromagnetic radiations is fundamentally dependent on the availability of facilities capable of generating suitable testing scenarios in a reproducible and controllable manner. The most classical example is certainly the case of a locally-plane wave, typically assumed to propagate within an anechoic environment to simulate a free-space configuration. A number of solutions have been developed in the past, giving rise to such facilities as openarea test sites, compact ranges, TEM cells, and the like. All of these available solutions are somehow based on efforts to simulate an anechoic environment, a task often achieved by means of anechoic chambers (ACs), which rely on the use of electromagnetic absorbing materials.

Among the several reasons for choosing this type of environment is the simplicity of interpretation of the results of

A. Cozza is with the Département de Recherche en Électromagnétisme, Laboratoire des Signaux et Systèmes (L2S), UMR 8506 SUPELEC - Univ Paris-Sud - CNRS, 3 rue Joliot-Curie, 91192 Gif-sur-Yvette, France. Contact e-mail: andrea.cozza@supelec.fr.

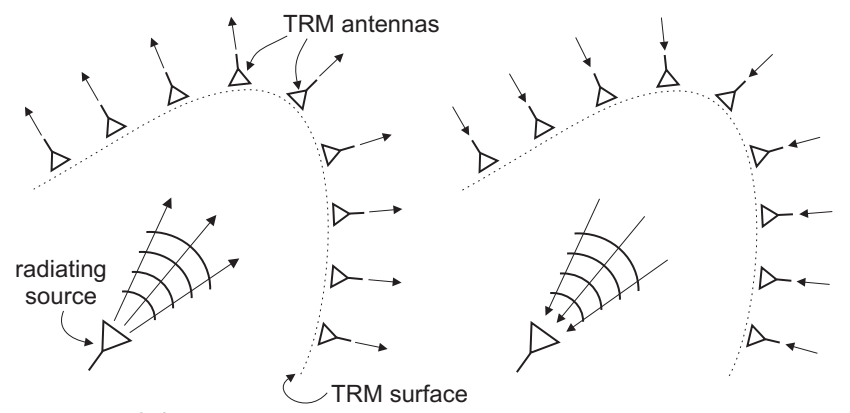

(a)

(b)
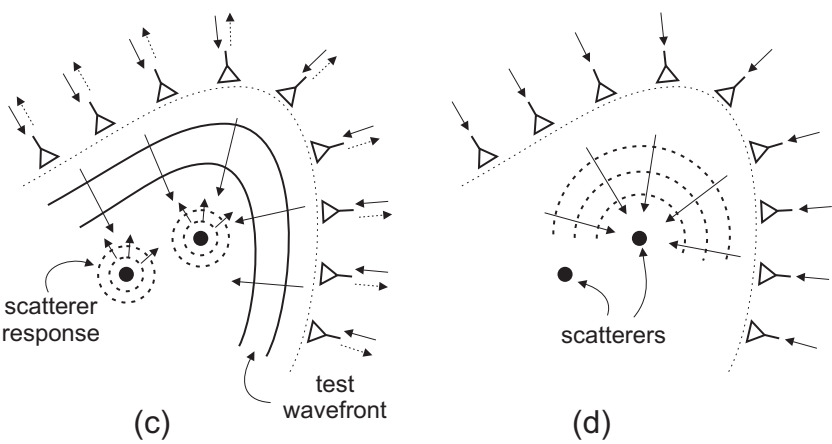

(d)

Fig. 1: A schematic representation of the two main TR techniques currently available: (a)-(b) TR of a radiating source; (c)-(d) selective focusing over a point scatterer by means of the DORT approach.

a test: having made use, at least ideally, of a single plane wave, the field scattered by the EUT, the currents induced over its external surface or at its interior are all straightforwardly linked to a single and well-defined external excitation.

Such an approach shows its limitations as soon as a large number of testing configurations is required (changing direction of arrival, polarization, etc.), thus leading to the need of complex and time-consuming mechanical displacements of the source or of the EUT. Albeit light-weight antennas and EUTs can be easily moved around, the case of large EUTs such as those considered in the aerospace industry (e.g., satellites, airplanes) or in electromagnetic compatibility (e.g., vehicles) requires complex mechanical solutions. A class of testing configurations in itself where a similar problem is observed is that involving the emulation of complex propagation environments, such as for telecommunication tests: the need to reproduce multi-path or fading environments comes with 
expensive solutions in ACs [1], [2].

For all these reasons, reverberation chambers (RCs) have gained a wide following even outside the electromagnetic compatibility (EMC) community, especially for the need of assessing the performances of telecommunication devices for data-transmission schemes through complex environments [3]. Here, the logic of the test is turned upside-down: the testing conditions do not require anymore moving the sources or the EUT, since it is considered that RCs can provide a testing scenario where a large number of plane waves propagate along ideally all possible directions [4], [5], [6]. The well-known price to pay for this simplification is the loss of intuitive understanding of the undergoing physical phenomena leading to the test results, and the important issue of having hardly repeatable testing conditions. We acknowledge the fact that the average testing conditions are repeatable, but the exact configuration is actually not.

As opposed to these two scenarios, in the last few years it has been shown that the preconceived idea of reverberating cavities as capable of supporting only narrow-band excitations and incoherent fields should be revisited. Time-reversal (TR) techniques have been shown to be capable of generating coherent wavefronts that can behave in a similar way to anechoic environments [7], [8], [9]. The availability of such a new way of using RCs is particularly exciting because it could be a way of accessing the main features of RCs and ACs at the same time, within the same facility, by taking advantage of a high energy efficiency while being able to generate simpler and more easily predictable wavefronts. Unfortunately, as we argue in the next two sections, currently available TR techniques are unsuitable for testing purposes, since they rely on a fixed two-step procedure that does not allow straightforwardly controlling the features of the generated wavefronts.

A more powerful technique disposing of these limitations was introduced in [10] and experimentally validated in [8]. It is based on the use of synthetic sources, leading to a generalized technique for the generation of time-reversed wavefronts whose features can be controlled in a very simple manner. In this paper, we present a formal analysis of how the equivalence theorem, coupled to a phase conjugation technique allows generating arbitrary wavefronts within random propagation media characterized by weak spatial correlation. Our theory is first introduced for a general medium, requiring only linearity and reciprocity, while in a second time we focus on the specific case of a medium supporting a diffused field distribution, e.g., an overmoded reverberation chamber. The dyadic operator describing how a target wavefront will be modified on average by the proposed technique is derived, and numerical examples are provided to illustrate our results. No experimental result is provided, since they are already available in the literature [8], [11].

As opposed to previous works dealing with the focusing of time-reversed waves in complex media [9], the proposed method is not limited by the intrinsical inability of standard TR techniques to generate arbitrary wavefronts. A major result is the proof that reverberation chambers are not only capable of generating wideband pulsed fields, but in a more general way to generate in an accurate way arbitrary coherent wavefronts, through a simple procedure. Our analysis leads to the conclusion that a paradigm shift can be introduced in TR applications when dealing with reverberation chambers (or more generally with wave-diffusive media), since the parameters defining the wavefront can be changed in real-time by means of standard signal-processing techniques, thus introducing the possibility of high-speed testing in reverberation chambers and the generation of complex, but deterministic, propagation scenarios.

\section{TIME-REVERSED WAVEFRONTS}

In this section we do not pretend to provide a thorough summary of TR, nor of all of its applications. A panoramic view of available TR applications is necessary in order to get a better grasp of the advances proposed in this paper.

TR is fundamentally the same technique previously known as phase conjugation, which originated in optics in the late 70's [12], primarily intended to compensate distortions (selfhealing) in wavefronts propagating through complex media, particularly with the aim of focusing energy towards a given position in space. All of the applications of TR are based on the TR symmetry of Helmholtz equation, implying an invariance of its solutions to a change of sign in the time variable.

TR applications typically exploit this property by coupling it to Huygens' principle: as depicted in Figs. 1(a)-(b), we can define a two-step procedure where the first step involves a source of radiation generating a diverging wavefront recorded by an ideally continuous set of transducers (e.g., antennas) deployed over a closed surface $\Sigma$. These transducers are usually referred to, in the context of TR applications, as a TR mirror (TRM) [13]. Coupling Huygens' principle to the TR symmetry of Helmholtz equation implies that by exciting the transducers with the time-reversed version of the signals received during the first phase, the TRM will generate an ideally perfect replica of the original wavefront, but this time converging back at the source, as a consequence of our inverting the direction of evolution of the time variable [13].

An important step in our proposal is the passage from the usually open media addressed when using the paradigm we just recalled, towards bounded ones, e.g., closed cavities. This issue was studied in several papers, e.g., [14], [15], [16], [11], where it was shown that the presence of reflective boundaries allows reducing the number of TRM transducers to just a few, typically one: this number is to be compared with the inevitably higher number of sources needed in wavefront synthesis in free-space environments [1], [2], a direct consequence of the spatial-sampling theorem [17]. The use of TR signals also allows the generation of short pulses within a reverberation chamber, which is of practical interest when testing EUTs closely exposed to high-power radar pulses [18].

The second approach to generating TR wavefronts is the DORT technique [19], [20]: while standard TR considers that what will be the target of the focusing wave during the second phase (Fig. 1(b)), needs to be a source during the first one (Fig. 1(a)), the DORT allows avoiding the target to be a source, whenever it behaves as a point scatterer, i.e., as a passive device that will respond with a spherical 
wavefront (Fig. 1(d)) to an externally excited locally plane wave (Fig. 1(c)). The DORT may appear to have a potential for testing applications, particularly when dealing with passive EUTs that cannot be operated as active sources. In fact, as we argue in the next section, the DORT could hardly be applied for testing purposes.

The problem with all of these methods is that in the available literature TR applications always aim at producing a focusing wavefront at some position in space. The motivation is never the generation of a wavefront per se: the reason why focusing is under consideration is typically either a clearer transmission of signal through complex media at a given position (e.g., a receiver in sonar [21] or telecommunication schemes [22]) or to improve imaging techniques [19], [20], [23]. As we will argue in the next section, this is not compatible with EUT testing, since EUTs are often electrically large and present distributed scattering features rather than localized ones.

\section{TR FROM A TESTING POINT OF VIEW}

Following this short discussion about current TR techniques, one may think that it could be interesting to implement them within RCs for at least two reasons: 1) to provide a solution to the problem of pulsed field generation; 2) as a way of more effectively generating high-intensity fields within a reverberation chamber. Such points are apparently useful only in the context of EMC, where the absolute intensity of the testing wavefront is of paramount importance. As it will be shown in the rest of this paper, the proposed generalized approach provides a more powerful rationale for the idea of coupling TR to reverberation chambers; as a matter of fact, a further motivation is the ability to control the generated wavefront without any mechanical displacement of the sources, nor any need for complex sources.

Before passing to the advantages brought in by our method, let us us start by looking at the shortcomings of TR from a testing point of view. If the standard paradigm depicted in Figs. 1(a)-(b) were used, how to generate in the first place the diverging wavefront (first phase) that will be time-reversed in order to be focused over the EUT? The eventual solution of applying auxiliary sources over the EUT in order to radiate the first-phase wavefront are bound to fail, since the standard paradigm would require passing through the two phases we have recalled in the previous section: hence, as soon as a new direction of incidence is to be established, the auxiliary sources would need to be moved over the EUT, and a new cycle of test would start all over. This is hardly acceptable, as it would require an increased number of manipulations with respect to tests carried out in anechoic chambers.

A potential solution could be envisaged by recalling the DORT paradigm (Figs. 1(c)-(d)): in this case, it would not be possible to chose whatever direction of incidence on the EUT, as the DORT can merely select a wavefront among the scattering responses of the EUT. If this response is (as often is the case) dominated by a few bright points [24], the choice of the test wavefront will be limited to the intrinsical response of the EUT, rather than satisfying the need to identify the

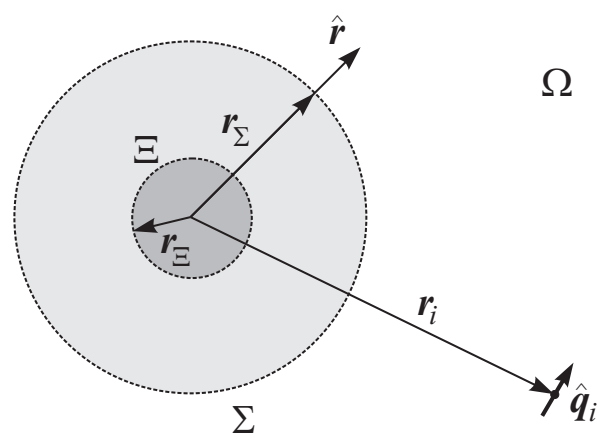

Fig. 2: Configuration for the application of Love's equivalence theorem. Equivalent electric and magnetic currents are defined over the surface $\Sigma$, representing the wavefront $\mathcal{E}_{\text {wf }}(\boldsymbol{r}, t)$ that would have been generated by a synthetic source contained in the volume bounded by the surface $\Xi$. These elements are embedded into a complex medium $\Omega$.

responses of the EUT to a varying, but predefined, testing wavefront.

The problem is that TR techniques in their present state are not suitable for EUT testing: they are actually mismatched to practical needs, as they have been designed to deal with mainly point scatterers, rather than electrically extended ones, as it is often the case when dealing with real-life EUTs, and this goes without taking into account the issue of polarization, which leads to an even more complex scenario when compared to the scalar-wave propagation and scattering undergoing in acoustics.

The solution to this mismatch is to shift our attention from the idea of focusing over a point to the idea of generating a controllable wavefront. By this last term, we consider the ability to control all of the parameters defining a wavefront, e.g., its time-dependence, polarization, directivity and direction of arrival. This reflection has motivated our proposing an alternative approach based on the use of synthetic sources (section IV), leading to a new paradigm for TR that is not only suitable for EMC purposes but also brings in new advantages for any test based on submitting an EUT to impinging wavefronts. This approach, that we have named the Time-Reversal Electromagnetic Chamber (TREC) was originally introduced in [10] while the first experimental validation was proposed in [8].

\section{A Generalized TR TECHNIQUE}

Our analysis takes its start from the standard two-step approach recalled in section II. The application we envision is peculiar in the sense that we do not consider the usual retrieval of the wavefront generated by an elementary source, but rather a generic wavefront. In this respect, we introduce the function $\mathcal{E}_{\mathrm{wf}}(\boldsymbol{r}, t)$ describing the space-time dependence of the wavefront we aim at generating, i.e., the target wavefront: in fact, $\mathcal{E}_{\mathrm{wf}}(\boldsymbol{r}, t)$ is the diverging wavefront that would be generated in a free-space environment, whereas the TREC will rather be used in order to deliver, ideally, $\mathcal{E}_{\mathrm{wf}}(\boldsymbol{r},-t)$, i.e., the converging version of the wavefront, used to test the EUT response in a number of potential applications. We are not 
interested in specifying the nature of the source generating the original $\mathcal{E}_{\text {wf }}(\boldsymbol{r}, t)$ wavefront, nor is it necessary: the wavefront $\mathcal{E}_{\mathrm{wf}}(\boldsymbol{r}, t)$ can be imagined as the result of an unspecified source, to be eventually found within a volume enclosed by the surface $\Xi$, introduced in Fig. 2. This surface will be assumed to be spherical for simplicity, with radius $r_{\Xi}$. Knowledge of the electrical dimension $r_{\Xi} / \lambda$ provides a direct measure of the potential directivity of the wavefronts radiated by the source [25]. For reasons that will be clearer at the end of this section, we will refer to this source as the synthetic source.

The divergent wavefront thus radiated during the first phase can be regarded through the lens of Love's equivalence theorem [26]: by defining a closed surface $\Sigma$ bounding the synthetic source (see Fig. 2), the sampling of the function $\mathcal{E}_{\mathrm{wf}}(\boldsymbol{r}, t)$ over $\Sigma$ allows defining equivalent currents capable of exactly reproducing the same space-time dependence at any position outside $\Sigma$ itself, independently from the eventual presence of an EUT.

Two assumptions will be introduced in order to simplify our analysis, with no loss of generality: the first one consists in regarding $\mathcal{E}_{\mathrm{wf}}(\boldsymbol{r}, t)$ over $\Sigma$ as the far-field radiation of the synthetic source, while the second one is to assume that $\Sigma$ be a spherical surface. The rationale for requiring a farfield radiation is twofold: first, our analysis will be greatly simplified, thanks to the simpler relationship existing between electric and magnetic fields, while the fact that time-reversed wavefronts are deprived of reactive components calls for the need to remove the reactive components off a wavefront before comparing it to the one generated at the end of the TR procedure, since only propagative components are conserved, as recalled in section II. The use of far-field wavefronts allows for a direct comparison of the target wavefront and the one actually generated by the proposed procedure.

According to these assumptions

$$
\mathcal{H}_{\mathrm{wf}}(\boldsymbol{r}, t)=\frac{1}{\zeta_{0}} \hat{\boldsymbol{r}} \times \mathcal{E}_{\mathrm{wf}}(\boldsymbol{r}, t) \quad \boldsymbol{r} \in \Sigma,
$$

where $\zeta_{0}$ is the free-space wave impedance and $\hat{\boldsymbol{r}}$ is the radial unit vector coinciding with the outward pointing unit vector normal to $\Sigma$, as depicted in Fig. 2. As we are dealing with the generation of arbitrary wavefronts, and in particular pulsed ones, a time-domain description should be the final outcome of our analysis. Still, the intermediary steps of our analysis will be carried out in the frequency domain. We thus introduce the wavefront description for the electric field $\boldsymbol{E}_{\mathrm{wf}}(\boldsymbol{r}, \omega)$ in Fourier frequency domain, defined as

$$
\boldsymbol{E}_{\mathrm{wf}}(\boldsymbol{r}, \omega)=\mathcal{F}\left\{\mathcal{E}_{\mathrm{wf}}(\boldsymbol{r}, t)\right\},
$$

where $\mathcal{F}\{\cdot\}$ is Fourier transform, with the magnetic-field spectrum similarly defined.

The equivalent electric and magnetic currents over $\Sigma$ can thus be defined as

$$
\begin{aligned}
\boldsymbol{J}_{e}(\boldsymbol{r}, \omega) & =\boldsymbol{J}_{e}^{\prime}(\boldsymbol{r}, \omega) \delta\left(\boldsymbol{r}-\boldsymbol{r}_{\Sigma}\right) \\
\boldsymbol{J}_{m}(\boldsymbol{r}, \omega) & =\boldsymbol{J}_{m}^{\prime}(\boldsymbol{r}, \omega) \delta\left(\boldsymbol{r}-\boldsymbol{r}_{\Sigma}\right),
\end{aligned}
$$

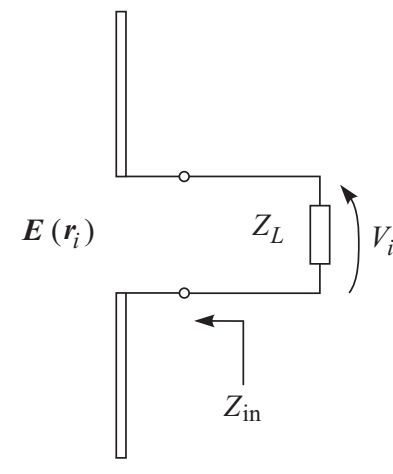

(a)

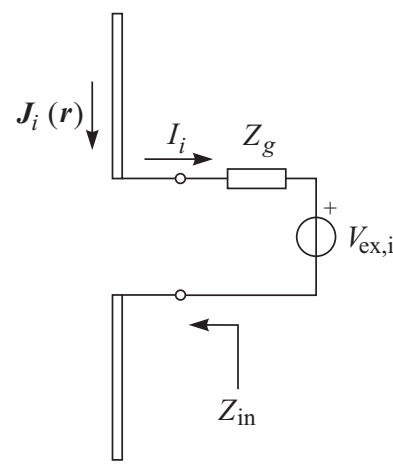

(b)
Fig. 3: Equivalent models of the TRM antennas used for the computation of the signals received during the first standard TR phase and the field generated by them in the second phase: (a) receiving-mode model; (b) transmission-mode model.

with

$$
\begin{aligned}
\boldsymbol{J}_{e}^{\prime}(\boldsymbol{r}, \omega) & =-\frac{\boldsymbol{E}_{\mathrm{wf}}(\boldsymbol{r}, \omega)}{\zeta_{0}} \\
\boldsymbol{J}_{m}^{\prime}(\boldsymbol{r}, \omega) & =\boldsymbol{E}_{\mathrm{wf}}(\boldsymbol{r}, \omega) \times \hat{\boldsymbol{r}} .
\end{aligned}
$$

where $\boldsymbol{r}_{\Sigma}$ is a vector spanning the surface $\Sigma$ and $\delta(\boldsymbol{r})$ is Dirac's delta distribution.

Given the distributions of electric and magnetic currents, the electric field $\boldsymbol{E}(\boldsymbol{r}, \omega)$ they generate at any position within a propagation medium can be expressed by means of its dyadic Green's functions as

$$
\begin{aligned}
& \boldsymbol{E}(\boldsymbol{r}, \omega)=\int_{\Sigma} \tilde{\boldsymbol{G}}_{\mathrm{ee}}\left(\boldsymbol{r}, \boldsymbol{r}^{\prime}, \omega\right) \cdot \boldsymbol{J}_{e}^{\prime}\left(\boldsymbol{r}^{\prime}, \omega\right) \mathrm{d}^{2} \boldsymbol{r}^{\prime}+ \\
& \int_{\Sigma} \tilde{\boldsymbol{G}}_{\mathrm{em}}\left(\boldsymbol{r}, \boldsymbol{r}^{\prime}, \omega\right) \cdot \boldsymbol{J}_{m}^{\prime}\left(\boldsymbol{r}^{\prime}, \omega\right) \mathrm{d}^{2} \boldsymbol{r}^{\prime} .
\end{aligned}
$$

where the two dyadic functions $\tilde{\boldsymbol{G}}_{\mathrm{ee}}\left(\boldsymbol{r}, \boldsymbol{r}^{\prime}, \omega\right)$ and $\tilde{\boldsymbol{G}}_{\mathrm{em}}\left(\boldsymbol{r}, \boldsymbol{r}^{\prime}, \omega\right)$ refer to the Green's functions relating, respectively, electric and magnetic currents to the electric field.

The configuration depicted in Fig. 2 presents TRM antennas assimilable to elementary dipoles, positioned at $\boldsymbol{r}_{i}$ and oriented along $\hat{\boldsymbol{q}}_{i}$. These antennas will be operated in receiving and transmitting mode and can thus be described by means of the equivalent models shown in Fig. 3. In this framework, the electric field $\boldsymbol{E}\left(\boldsymbol{r}_{i}, \omega\right)$ related to the wavefront will eventually couple with them, leading to an output voltage $V_{i}(\omega)$

$$
V_{i}(\omega)=\frac{Z_{L}(\omega)}{Z_{L}(\omega)+Z_{\text {in }}(\omega)} \boldsymbol{E}\left(\boldsymbol{r}_{i}, \omega\right) \cdot \boldsymbol{h}_{e, i}(\omega),
$$

where $\boldsymbol{h}_{e, i}(\omega)$ is the vector effective height of the TRM antennas

$$
\boldsymbol{h}_{e, i}(\omega)=h_{e}(\omega) \hat{\boldsymbol{q}}_{i},
$$

having assumed all of the antennas to have an identical effective height $h_{e}(\omega)$.

As recalled in section II, TR applications require the output signals $V_{i}(\omega)$ to be time-reversed, or phase-conjugated in the frequency domain, and subsequently applied to the TRM 
antennas. The TR of the $V_{i}(\omega)$ output signals would naturally require considering a delay $T$ representing the duration of the first phase during which the output signals are recorded. This delay will be neglected in the rest of the paper, as it only leads to a phase-shift term shared by all of the output signals, with no impact on the results.

During the second phase of TR, the TRM antennas will be excited by means of the signals $V_{\text {ex }, \mathrm{i}}(\omega)=\bar{V}_{i}(\omega)$, where the overhead bar stands for phase conjugation. According to the equivalent model in Fig. 3(b), the antennas will be driven by a current $I_{i}(\omega)$

$$
I_{i}(\omega)=-\frac{V_{\mathrm{ex}, \mathrm{i}}(\omega)}{Z_{g}(\omega)+Z_{\mathrm{in}}(\omega)},
$$

leading to an equivalent electric-current density

$$
\boldsymbol{J}_{i}\left(\boldsymbol{r}_{i}, \omega\right)=I_{i}(\omega) \boldsymbol{h}_{e}(\omega) \delta\left(\boldsymbol{r}-\boldsymbol{r}_{i}\right) .
$$

The electric field thus generated by each TRM antenna during the emission phase can be expressed as

$$
\begin{aligned}
\boldsymbol{E}_{\mathrm{TR}, \mathrm{i}}(\boldsymbol{r}, \omega) & =\int_{\Omega} \tilde{\boldsymbol{G}}_{\mathrm{ee}}\left(\boldsymbol{r}, \boldsymbol{r}^{\prime}, \omega\right) \cdot \boldsymbol{J}_{i}\left(\boldsymbol{r}^{\prime}, \omega\right) \mathrm{d}^{3} \boldsymbol{r}^{\prime} \\
& =h_{e}(\omega) I_{i}(\omega) \tilde{\boldsymbol{G}}_{\mathrm{ee}}\left(\boldsymbol{r}, \boldsymbol{r}_{i}, \omega\right) \cdot \hat{\boldsymbol{q}}_{i} .
\end{aligned}
$$

In order to simplify our notations, we introduce the electric and magnetic vector transfer functions

$$
\begin{aligned}
\boldsymbol{N}_{e, i}(\boldsymbol{r}, \omega) & =\tilde{\boldsymbol{G}}_{\mathrm{ee}}\left(\boldsymbol{r}, \boldsymbol{r}_{i}, \omega\right) \cdot \hat{\boldsymbol{q}}_{i} \\
\boldsymbol{N}_{m, i}(\boldsymbol{r}, \omega) & =\tilde{\boldsymbol{G}}_{\mathrm{em}}\left(\boldsymbol{r}, \boldsymbol{r}_{i}, \omega\right) \cdot \hat{\boldsymbol{q}}_{i},
\end{aligned}
$$

relating the electric or magnetic field observed at a generic position $r$ to the signals applied to the input port of the $i$-th TRM antenna. They have units of $\Omega \mathrm{m}^{-2}$ and $\mathrm{m}^{-2}$, respectively.

Inserting (5)-(8) into (10), and making use of (11), yields

$$
\begin{aligned}
& \boldsymbol{E}_{\mathrm{TR}, \mathrm{i}}(\boldsymbol{r}, \omega)= \\
& -C(\omega)\left\{\int_{\Sigma} \boldsymbol{N}_{e, i}(\boldsymbol{r}, \omega) \overline{\boldsymbol{N}}_{e, i}\left(\boldsymbol{r}^{\prime}, \omega\right) \cdot \overline{\boldsymbol{J}}_{e}^{\prime}\left(\boldsymbol{r}^{\prime}, \omega\right) \mathrm{d}^{2} \boldsymbol{r}^{\prime},\right. \\
& \left.+\int_{\Sigma} \boldsymbol{N}_{e, i}(\boldsymbol{r}, \omega) \overline{\boldsymbol{N}}_{m, i}\left(\boldsymbol{r}^{\prime}, \omega\right) \cdot \overline{\boldsymbol{J}}_{m}^{\prime}\left(\boldsymbol{r}^{\prime}, \omega\right) \mathrm{d}^{2} \boldsymbol{r}^{\prime}\right\}
\end{aligned}
$$

having exploited the spatial reciprocity of Green's functions, and thus of the vector transfer functions (11). The quantity $C(\omega)$ in (12) takes care of the electrical parameters of the TRM antennas

$$
C(\omega)=\frac{\left|h_{e}(\omega)\right|^{2}}{Z_{g}(\omega)+Z_{\text {in }}(\omega)} \frac{\bar{Z}_{L}(\omega)}{\bar{Z}_{L}(\omega)+\bar{Z}_{\text {in }}(\omega)} .
$$

It is noteworthy that the doublets of vector transfer functions under the integral signs are dyadic functions. Equivalent currents (4) can now be inserted, leading to

$$
\begin{aligned}
& \boldsymbol{E}_{\mathrm{TR}, \mathrm{i}}(\boldsymbol{r}, \omega)=\frac{1}{\zeta_{0}} \int_{\Sigma} \boldsymbol{N}_{e, i}(\boldsymbol{r}, \omega) \overline{\boldsymbol{N}}_{e, i}\left(\boldsymbol{r}^{\prime}, \omega\right) \cdot \overline{\boldsymbol{E}}_{\mathrm{wf}}\left(\boldsymbol{r}^{\prime}, \omega\right) \mathrm{d}^{2} \boldsymbol{r}^{\prime}+ \\
& -\int_{\Sigma} \boldsymbol{N}_{e, i}(\boldsymbol{r}, \omega) \hat{\boldsymbol{r}}^{\prime} \times \overline{\boldsymbol{N}}_{m, i}\left(\boldsymbol{r}^{\prime}, \omega\right) \cdot \overline{\boldsymbol{E}}_{\mathrm{wf}}\left(\boldsymbol{r}^{\prime}, \omega\right) \mathrm{d}^{2} \boldsymbol{r}^{\prime}
\end{aligned}
$$

having assumed excitation signals $V_{\mathrm{ex}, \mathrm{i}}(\omega)=\bar{V}_{i}(\omega) / C(\omega)$.

This last result allows linking the space-time description of the target wavefront to the one actually generated after
TR excitations are applied to the TRM antennas. Since we are interested in wave-diffusive media, the vector transfer functions (11) will be considered as random functions in section VI. The self-averaging property of TR [27], [28] implies that the wavefronts generated by a TREC converge towards their ensemble average, independently from the random realizations of Green's dyadic functions. This issue is discussed in Appendix B.

Hence, we introduce the average dyadic responses

$$
\begin{gathered}
\tilde{\boldsymbol{T}}_{\mathrm{ee}, \mathrm{i}}\left(\boldsymbol{r}, \boldsymbol{r}^{\prime}, \omega\right)=\mathrm{E}\left[\boldsymbol{N}_{e, i}(\boldsymbol{r}, \omega) \overline{\boldsymbol{N}}_{e, i}\left(\boldsymbol{r}^{\prime}, \omega\right)\right] \\
\tilde{\boldsymbol{T}}_{\mathrm{em}, \mathrm{i}}\left(\boldsymbol{r}, \boldsymbol{r}^{\prime}, \omega\right)= \\
\mathrm{E}\left[\boldsymbol{N}_{e, i}(\boldsymbol{r}, \omega)\left(\hat{\boldsymbol{r}}^{\prime} \times \overline{\boldsymbol{N}}_{m, i}\left(\boldsymbol{r}^{\prime}, \omega\right)\right)\right] \\
\tilde{\boldsymbol{T}}_{\mathrm{i}}\left(\boldsymbol{r}, \boldsymbol{r}^{\prime}, \omega\right)= \\
\frac{1}{\zeta_{0}} \tilde{\boldsymbol{T}}_{\mathrm{ee}, \mathrm{i}}\left(\boldsymbol{r}, \boldsymbol{r}^{\prime}, \omega\right)-\tilde{\boldsymbol{T}}_{\mathrm{em}, \mathrm{i}}\left(\boldsymbol{r}, \boldsymbol{r}^{\prime}, \omega\right)
\end{gathered}
$$

with $E[\cdot]$ the ensemble-average operator. These functions are directly related to the autocorrelation functions of the generic random process $\boldsymbol{N}(\boldsymbol{r}, \omega)$ and are anisotropic. Finally, (14) gives place to

$$
\mathrm{E}\left[\boldsymbol{E}_{\mathrm{TR}, \mathrm{i}}(\boldsymbol{r}, \omega)\right]=\int_{\Sigma} \tilde{\boldsymbol{T}}_{\mathrm{i}}\left(\boldsymbol{r}, \boldsymbol{r}^{\prime}, \omega\right) \cdot \overline{\boldsymbol{E}}_{\mathrm{wf}}\left(\boldsymbol{r}^{\prime}, \omega\right) \mathrm{d}^{2} \boldsymbol{r}^{\prime},
$$

requiring no assumption on the nature of the medium, nor on the type of wavefront distribution, apart the simplifying assumption of a far-field configuration.

The possibility of obtaining an accurate transmission through a complex medium is feasible in the special case of a diffusive medium, which is characterized by a low spatialcorrelation, thus ensuring an equivalent Green's function close to that of a free-space environment (see section VI). The fundamental point that we want to stress here is that the application of a TR approach allows reproducing a behavior that is actually closer to a free-space environment, but within a complex medium supporting a diffused-field configuration. This idea is illustrated in section VIII.

The far-field assumption was introduced as a way of simplifying the derivation of the above results, but the equivalence theorem is not affected by the region of radiation of a source, and will stay exact even in its reactive region, even though only the propagative part of the target wavefront will be reproduced, thus leading to the inevitable diffraction limit in the focus region [29].

\section{A PARADIGM SHIFT FOR TR APPLICATIONS}

The derivation of (16) implies that as soon as the dyadic Green's functions of the medium are known between the points over $\Sigma$ and the positions of the TRM antennas, a direct relationship can be promptly established between the target wavefront distribution and the one generated by the TREC.

Moreover, the derivation leading to (16) paves the way for a change of paradigm in the use of TR techniques: indeed, as soon as the vector transfer functions $\boldsymbol{N}_{e, i}(\boldsymbol{r}, \omega)$ and $\boldsymbol{N}_{r, i}(\boldsymbol{r}, \omega)$ are known, it is no more necessary to undergo the two standard phases of TR. The signals $V_{i}(\omega)$ that would 


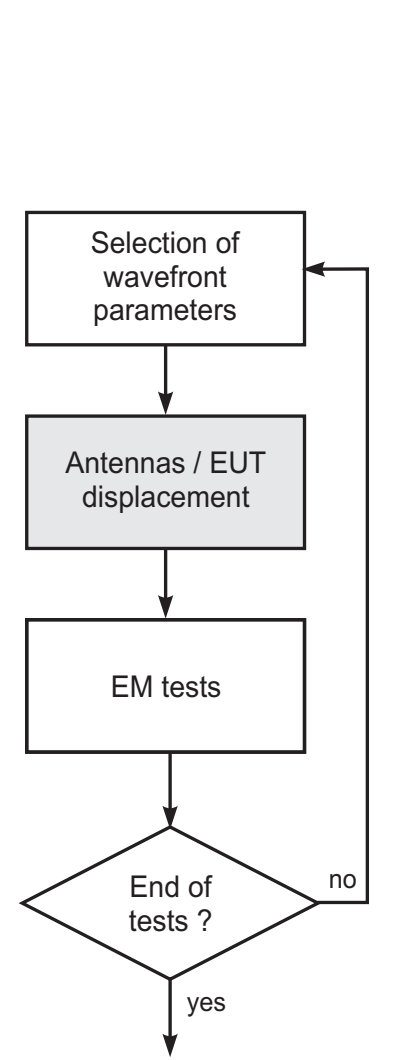

(a)

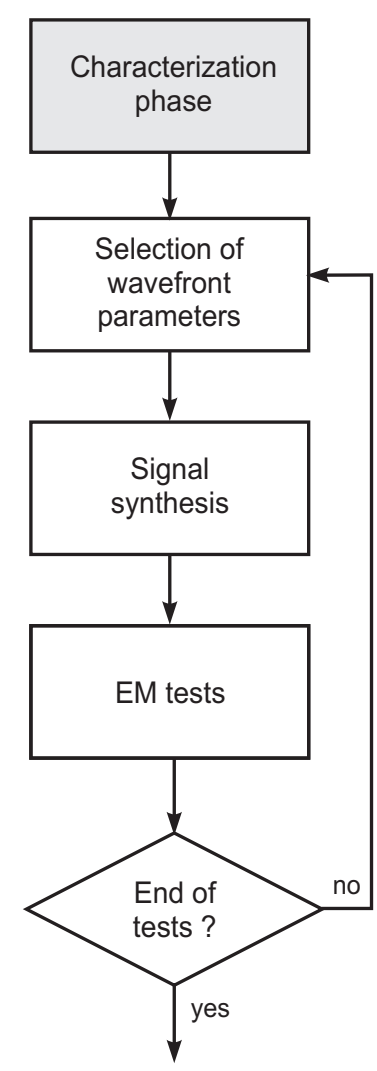

(b)
Fig. 4: Sequences of necessary steps to follow when using an (a) anechoic test environment or a (b) TREC. Shaded blocks represent operations based on mechanical displacements or substitution of devices.

result from the recording phase (or first phase) can be straightforwardly computed for any target wavefront without needing its actually being radiated, since

$$
V_{i}(\omega) \propto \int_{\Sigma} \boldsymbol{N}_{\mathrm{eq}, i}(\boldsymbol{r}, \omega) \cdot \boldsymbol{E}_{\mathrm{wf}}(\boldsymbol{r}, \omega) \mathrm{d}^{2} \boldsymbol{r},
$$

with

$$
\boldsymbol{N}_{\mathrm{eq}, i}(\boldsymbol{r}, \omega)=-\frac{\boldsymbol{N}_{e, i}(\boldsymbol{r}, \omega)}{\zeta_{0}}+\hat{\boldsymbol{r}} \times \boldsymbol{N}_{m, i}(\boldsymbol{r}, \omega),
$$

leading to a direct synthesis of the excitation signals for the second phase.

This simple modification has deep consequences: as a matter of fact, the standard implementation of TR techniques implies that each time that a new converging wavefront is to be generated, the diverging version of the same wavefront needs to be generated by an actual source during the first phase. Furthermore, as the characteristics of the wavefront change, e.g., the direction of arrival or the polarization, the first phase is to be carried out again. This is clearly a strong limitation when proposing TR for testing applications, since as soon as a wide range of configurations is to be tested, the repetition of the two phases would be too costly. Moreover, the question of how to generate the diverging wavefront in the first place is far from trivial. The paradigm shift we propose solves all of these problems in a elegant and simple way. In fact, a preliminary characterization or learning phase can be considered during which the propagation medium is explored, proceeding to a direct measurement of $\boldsymbol{N}_{\mathrm{eq}, i}(\boldsymbol{r}, \omega)$ without any need to formulate any hypothesis on its nature. At this point, the signals that would have been received for any wavefront can be directly computed by means of (17).

The use of (17) implies that once a physical target wavefront is defined, the excitation signals needed to apply to the TRM antennas are readily available, even though the generation of this same wavefront could prove to be difficult when using real-life sources. It is therefore appropriate to refer to the timereversed wavefronts provided by (17) as generated by synthetic sources. The approach here proposed is somewhat reminiscent of synthetic aperture radar techniques, where post-processing techniques allow to emulate the availability of a source that would be practically unfeasible, while its emulation is actually quite straightforward. The difference is that although the synthetic source does not radiate in the first place, its TR wavefront is actually generated, not only computed in a postprocessing fashion.

The advantages of this approach are clear: passing from one wavefront to another just involves the synthesis of new excitation signals, without any need for further measurements, as long as the propagation medium has not undergone any modification, e.g., due to mechanical displacements of the EUT. Moreover, the linearity of the propagation of waves within the medium implies that superposition of effects holds: as a consequence, it is possible to conceive complex test scenarios where multiple wavefronts can be generated to impinge onto the EUT from different directions, with any type of time-dependence associated to each individual wavefront. The generation of similar scenarios by means of state-of-theart facilities would involve a sophisticated system to feed the antennas associated to each direction of arrival and control their orientations [1]. As opposed to this need, the TREC is theoretically capable of generating arbitrary wavefronts with a reduced number of antennas, typically just one, by exploiting the weak spatial correlation of the wave-diffusive media [30].

From a practical point of view, this approach allows dramatically reducing the time needed for generating a new converging wavefront, as the only steps needed are the computation of the excitation signals and their direct digital synthesis. A flow-chart representation of the sequence of operations needed when testing with a standard anechoic chamber or a TREC based on an overmoded cavity is proposed in Fig. 4, where it is made clear that the modification of the testing wavefront does not require any physical modification of the test environment, but only changing excitation signals. Rather than repeating mechanical displacements each time that a new test configuration is required, these are relegated to the learning phase, before starting a cycle of uninterrupted tests.

The entire procedure here suggested relies on previous knowledge of the $\boldsymbol{N}_{\text {eq,i }}(\boldsymbol{r}, \omega)$ functions. A detailed discussion of this issue is out of the scope of this paper, and it was partially considered in [8]. 


\section{THE CASE OF A WAVE-DIFFUSIVE MEDIUM}

In this section we address the special case of an ideal wavediffusive medium. By this term we consider any medium, not necessarily homogeneous, whose Green's functions can be approximated by means of a superposition of a large number of random plane waves propagating with equal probability along any direction [31], ensuring very simple statistical properties for the field: a Gaussian-distributed field with spatial-invariant moments and a perfect depolarization. Among the several configurations where this property can be invoked, large cavities such as reverberation chambers are perhaps the simplest way of implementing it, as soon as an overmoded condition is satisfied [32], [33].

The hypothesis of perfect diffusion and thus a random plane-wave spectrum are actually the basis for the asymptotic analysis of reverberation chambers, as proposed, e.g., in [4]. As a direct consequence of spatial stationarity, $\tilde{\boldsymbol{T}}_{\mathrm{i}}\left(\boldsymbol{r}, \boldsymbol{r}^{\prime}, \omega\right)$ are independent from the position of the TRM antennas and their orientation, so that it is possible to drop the $i$ index and consider the average response $\tilde{\boldsymbol{T}}\left(\boldsymbol{r}, \boldsymbol{r}^{\prime}, \omega\right)$ of the TREC.

In order to derive a closed-form expression for the dyadic operator $\tilde{\boldsymbol{T}}\left(\boldsymbol{r}, \boldsymbol{r}^{\prime}, \omega\right)$, we expand the vector transfer functions (11) over the local reference system depicted in Fig. 5, defined by of a longitudinal unit vector $\hat{\rho}=d /\|d\|$, where $d=r^{\prime}-r$, a transversal unit vector $\hat{\boldsymbol{\nu}}$ lying on the plane defined by the vectors $\boldsymbol{r}$ and $\boldsymbol{r}^{\prime}$ and a third unit vector $\hat{\boldsymbol{\eta}}=\hat{\boldsymbol{\rho}} \times \hat{\boldsymbol{\nu}}$.

The dyadic operators introduced in (15), e.g., for the case of the $\tilde{\boldsymbol{T}}_{\text {ee }}\left(\boldsymbol{r}, \boldsymbol{r}^{\prime}, \omega\right)$ dyadic function, can thus be expressed into this new basis, yielding scalar components

$$
\begin{aligned}
& \left(\tilde{\boldsymbol{T}}_{\mathrm{ee}}\right)_{\hat{\boldsymbol{u}}_{m} \hat{\boldsymbol{u}}_{n}}\left(\boldsymbol{r}, \boldsymbol{r}^{\prime}, \omega\right)= \\
& \mathrm{E}\left[\hat{\boldsymbol{u}}_{m} \cdot \boldsymbol{N}_{\mathrm{e}}(\boldsymbol{r}, \omega) \hat{\boldsymbol{u}}_{n} \cdot \overline{\boldsymbol{N}}_{\mathrm{e}}\left(\boldsymbol{r}^{\prime}, \omega\right)\right],
\end{aligned}
$$

where $\hat{\boldsymbol{u}}_{m}$ is any of the basis unit vectors $\hat{\boldsymbol{\rho}}, \hat{\boldsymbol{\eta}}$ and $\hat{\boldsymbol{\nu}}$. Recalling that the vector functions $\boldsymbol{N}(\boldsymbol{r}, \omega)$ are generic transfer functions observed within a diffusive medium, the scalar terms (19) actually represent the covariances between the scalar components of two transfer functions evaluated at two positions within the medium. Hence, the results presented in [34] apply, leading to

$$
\begin{aligned}
& \left(\tilde{\boldsymbol{T}}_{\mathrm{ee}}\right)_{\hat{\boldsymbol{\rho}} \hat{\boldsymbol{\rho}}}\left(\boldsymbol{r}, \boldsymbol{r}^{\prime}, \omega\right)=\frac{N_{\mathrm{e}, \mathrm{av}}^{2}(\omega)}{3} \rho_{l}(d, \omega) \\
& \left(\tilde{\boldsymbol{T}}_{\mathrm{ee}}\right)_{\hat{\boldsymbol{\nu}} \hat{\boldsymbol{\nu}}}\left(\boldsymbol{r}, \boldsymbol{r}^{\prime}, \omega\right)=\frac{N_{\mathrm{e}, \mathrm{av}}^{2}(\omega)}{3} \rho_{t}(d, \omega) \\
& \left(\tilde{\boldsymbol{T}}_{\mathrm{ee}}\right)_{\hat{\boldsymbol{\eta}} \hat{\boldsymbol{\eta}}}\left(\boldsymbol{r}, \boldsymbol{r}^{\prime}, \omega\right)=\left(\tilde{\boldsymbol{T}}_{\mathrm{ee}}\right)_{\hat{\boldsymbol{\nu}} \hat{\boldsymbol{\nu}}}\left(\boldsymbol{r}, \boldsymbol{r}^{\prime}, \omega\right),
\end{aligned}
$$

results that hold for any wave-diffusive medium.

In (20), $N_{\mathrm{e}, \text { av }}(\omega) / \sqrt{3}$ is the rms amplitude of the electric field observed along any of its scalar components within the region of space where the ideal diffused-field conditions hold [35]; this quantity is derived in Appendix A. The functions $\rho_{t}(d, \omega)$ and $\rho_{l}(d, \omega)$ are spatial correlation functions, so they only depend on the distance $d=\left|\boldsymbol{r}-\boldsymbol{r}^{\prime}\right|$.

Apart for the three scalar components shown in (20), the remaining ones are identically null, as demonstrated in [34]. The two spatial-correlation functions $\rho_{l}(d, \omega)$ and $\rho_{t}(d, \omega)$

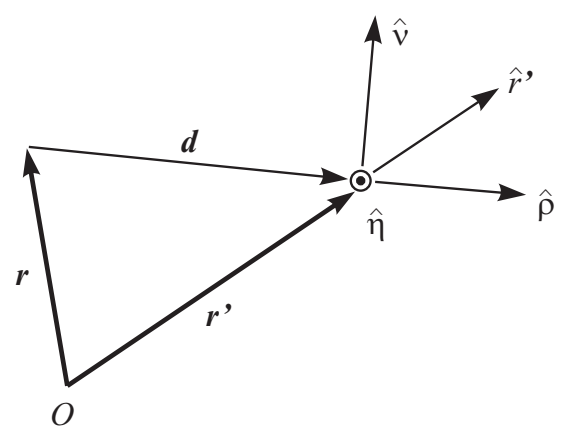

Fig. 5: The local reference system based on the orientation of the $r$ and $r^{\prime}$ vectors, defined by the right-hand set of unit vectors $\hat{\boldsymbol{\nu}}, \hat{\boldsymbol{\eta}}$ and $\hat{\boldsymbol{\rho}}$. This choice is at the basis of the results derived for the case of a wave-diffusive medium, e.g., an overmoded reverberation chamber.

refer to the longitudinal the transversal components of the electric field, respectively. These functions are [34]

$$
\begin{aligned}
\rho_{l}(d, \omega) & =\frac{3}{(k d)^{2}}[\operatorname{sinc}(k d)-\cos (k d)] \\
\rho_{t}(d, \omega) & =\frac{3}{2} \operatorname{sinc}(k d)-\frac{1}{2} \rho_{l}(d, \omega),
\end{aligned}
$$

with $k=\omega / c_{0}$ the wave-number and $c_{0}$ the speed of light in the homogeneous medium filling the cavity.

In dyadic formalism, $\tilde{\boldsymbol{T}}_{\text {ee }}\left(\boldsymbol{r}, \boldsymbol{r}^{\prime}, \omega\right)$ can be expressed as

$$
\begin{aligned}
& \tilde{\boldsymbol{T}}_{\mathrm{ee}}\left(\boldsymbol{r}, \boldsymbol{r}^{\prime}, \omega\right)= \\
& \quad \frac{N_{\mathrm{e}, \mathrm{av}}^{2}(\omega)}{3}\left[\hat{\boldsymbol{\rho}} \hat{\boldsymbol{\rho}} \rho_{l}(d, \omega)+(\hat{\boldsymbol{\nu}} \hat{\boldsymbol{\nu}}+\hat{\boldsymbol{\eta}} \hat{\boldsymbol{\eta}}) \rho_{t}(d, \omega)\right] .
\end{aligned}
$$

Following the same procedure for the $\tilde{\boldsymbol{T}}_{\mathrm{em}}\left(\boldsymbol{r}, \boldsymbol{r}^{\prime}, \omega\right)$ dyadic function, recalling that only the cross-transversal components (defined with respect to $\hat{\rho}$ ) of the electric and magnetic fields are correlated [34], we can state that the only non-zero components are

$$
\begin{gathered}
\left(\tilde{\boldsymbol{T}}_{\mathrm{em}}\right)_{\hat{\boldsymbol{\nu}} \hat{\boldsymbol{\nu}}}\left(\boldsymbol{r}, \boldsymbol{r}^{\prime}, \omega\right)= \\
\frac{N_{\mathrm{e}, \mathrm{av}}^{2}(\omega)}{3 \zeta_{0}} \rho_{m}(d, \omega) \hat{\boldsymbol{r}}^{\prime} \times \hat{\boldsymbol{\nu}} \cdot \hat{\boldsymbol{\eta}} \\
\left(\tilde{\boldsymbol{T}}_{\mathrm{em}}\right)_{\hat{\boldsymbol{\nu}} \hat{\boldsymbol{\rho}}}\left(\boldsymbol{r}, \boldsymbol{r}^{\prime}, \omega\right)= \\
\frac{N_{\mathrm{e}, \mathrm{av}}^{2}(\omega)}{3 \zeta_{0}} \rho_{m}(d, \omega) \hat{\boldsymbol{r}}^{\prime} \times \hat{\boldsymbol{\eta}} \cdot \hat{\boldsymbol{\rho}} \\
\left(\tilde{\boldsymbol{T}}_{\mathrm{em}}\right)_{\hat{\boldsymbol{\eta}} \hat{\boldsymbol{\eta}}}\left(\boldsymbol{r}, \boldsymbol{r}^{\prime}, \omega\right)=\left(\tilde{\boldsymbol{T}}_{\mathrm{em}}\right)_{\hat{\boldsymbol{\nu}} \hat{\boldsymbol{\nu}}}\left(\boldsymbol{r}, \boldsymbol{r}^{\prime}, \omega\right),
\end{gathered}
$$

where $\rho_{m}(d, \omega)$ is the mixed correlation function between the cross-transveral components of the electric and magnetic fields, given by [34]

$$
\rho_{m}(d, \omega)=-\frac{1}{2} \mathrm{j} k d \rho_{l}(d, \omega) .
$$

Whence, the operator $\tilde{\boldsymbol{T}}\left(\boldsymbol{r}, \boldsymbol{r}^{\prime}, \omega\right)$ reads, for the case of an ideally diffused field

$$
\tilde{\boldsymbol{T}}\left(\boldsymbol{r}, \boldsymbol{r}^{\prime}, \omega\right)=\frac{N_{\mathrm{e}, \mathrm{av}}^{2}(\omega)}{3 \zeta_{0}} \tilde{\boldsymbol{\rho}}\left(\boldsymbol{r}, \boldsymbol{r}^{\prime}, \omega\right)
$$



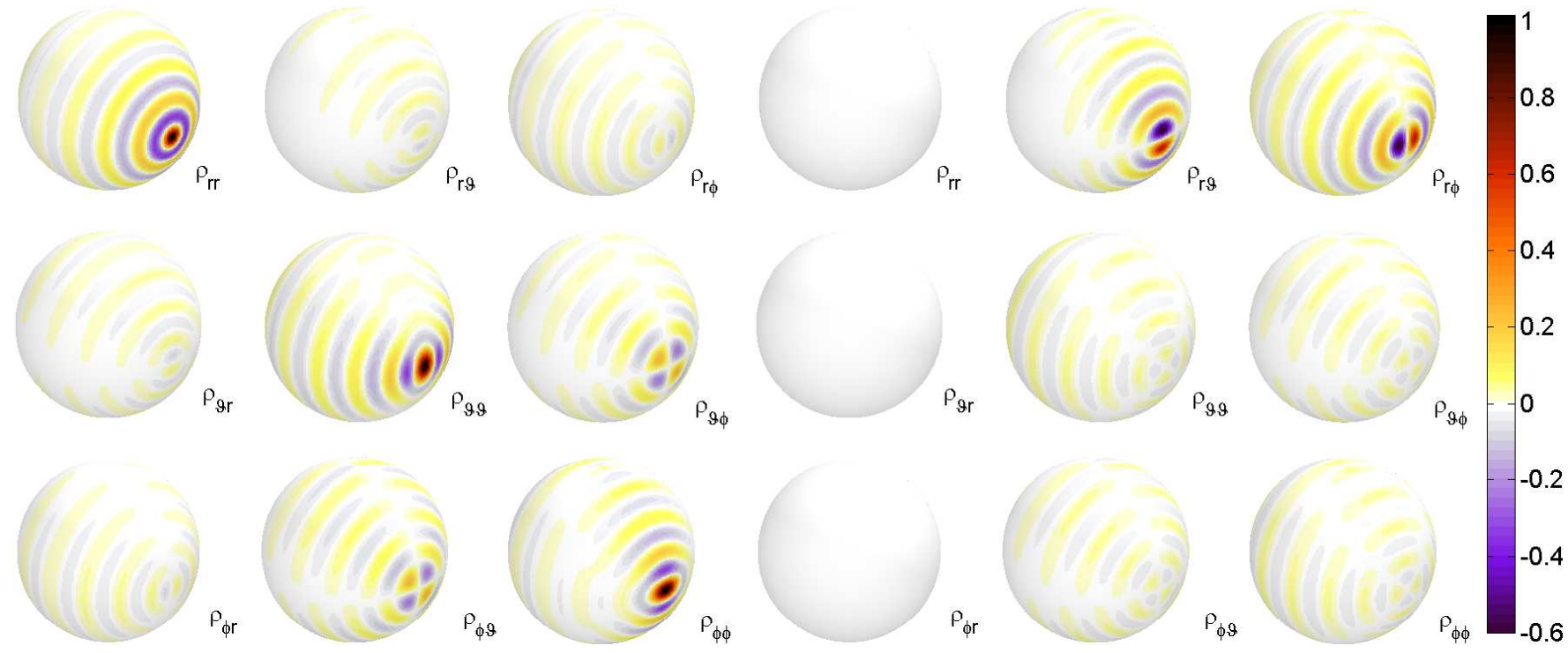

(a)

(b)

Fig. 6: Normalized dyadic function $\tilde{\boldsymbol{\rho}}\left(\boldsymbol{r}, \boldsymbol{r}^{\prime}, \omega\right)$ computed for $\boldsymbol{r} \in \Sigma$ and $\boldsymbol{r}^{\prime}=r_{\Sigma} \hat{\boldsymbol{x}}$, with $r_{\Sigma}=3 \lambda$ : (a) real and (b) imaginary parts. The 9 terms of the dyadic response are shown, matrix-wise, considering standard spherical unit vectors, following the order $\hat{\boldsymbol{r}}, \hat{\vartheta}$ and $\hat{\varphi}$, defined with respect to a polar axis vertically oriented.

with

$$
\begin{aligned}
& \tilde{\boldsymbol{\rho}}\left(\boldsymbol{r}, \boldsymbol{r}^{\prime}, \omega\right)=\hat{\boldsymbol{\rho}} \hat{\boldsymbol{\rho}} \rho_{l}(d, \omega)-\hat{\boldsymbol{\nu}} \hat{\boldsymbol{\rho}} \rho_{m}(d, \omega) \hat{\boldsymbol{r}}^{\prime} \times \hat{\boldsymbol{\eta}} \cdot \hat{\boldsymbol{\rho}}+ \\
& +(\hat{\boldsymbol{\nu}} \hat{\boldsymbol{\nu}}+\hat{\boldsymbol{\eta}} \hat{\boldsymbol{\eta}})\left[\rho_{t}(d, \omega)-\rho_{m}(d, \omega) \hat{\boldsymbol{r}}^{\prime} \times \hat{\boldsymbol{\nu}} \cdot \hat{\boldsymbol{\eta}}\right] .
\end{aligned}
$$

introducing the normalized dyadic response $\tilde{\boldsymbol{\rho}}(\boldsymbol{d}, \omega)$. As a result, the real and imaginary parts of the scalar components of this function are now bounded to one, since they correspond to the degree of coherence of the medium [36]. The operator $\tilde{\boldsymbol{\rho}}\left(\boldsymbol{r}, \boldsymbol{r}^{\prime}, \omega\right)$ behaves as a point-spread function (PSF).

The PSF $\tilde{\boldsymbol{\rho}}\left(\boldsymbol{r}, \boldsymbol{r}^{\prime}, \omega\right)$ will be used in two frameworks: 1) by setting $\boldsymbol{r}, \boldsymbol{r}^{\prime} \in \Sigma$, it allows assessing how a TREC-generated wavefront is distorted with respect to the target one in the farfield region; 2) with $\boldsymbol{r}^{\prime} \in \Sigma$ and a generic $\boldsymbol{r}$, it provides a direct access to the spatial evolution of a wavefront generated by the proposed method.

\section{ON THE PSF OF THE TREC}

Albeit the previous results hold for any diffusive medium, hereafter we will assume that this medium is an overmoded reverberation chamber, filled by a reciprocal and homogeneous medium surrounding the EUT. The target wavefront can be expressed, in the frequency domain, as

$$
\boldsymbol{E}_{\mathrm{wf}}(\boldsymbol{r}, \omega)=X(\omega) G(r, \omega) \boldsymbol{F}(\hat{\boldsymbol{r}}, \omega),
$$

where $\boldsymbol{F}(\hat{\boldsymbol{r}}, \omega)$ is the radiation pattern of the synthetic source, $G(r, \omega)$ is the far-field Green's scalar function of the medium

$$
G(r, \omega)=\frac{\mathrm{e}^{-\mathrm{j} k_{0} r}}{4 \pi r}
$$

and $X(\omega)$ is related to the excitation signal that would be used to drive the synthetic source. Such a factorized representation is made possible by the assumption of $\Sigma$ being in the farfield region of the synthetic source. Again we stress the fact that this choice is not a limitation, but just a simplifying assumption. The time-domain representation of $\mathcal{E}_{\mathrm{wf}}(\boldsymbol{r}, t)$ can thus be expressed as

$$
\mathcal{E}_{\mathrm{wf}}(\boldsymbol{r}, t)=\frac{x\left(t-r / c_{0}\right)}{4 \pi r} *_{t} \mathcal{F}^{-1}\{\boldsymbol{F}(\hat{\boldsymbol{r}}, \omega)\},
$$

where $*_{t}$ stands for the convolution integral applied to the time variable; this result holds for any $\boldsymbol{r}=r \hat{\boldsymbol{r}}$ in the far-field region of the synthetic source.

Introducing (27) into (16) while using (25) yields

$$
\mathrm{E}\left[\mathcal{E}_{\mathrm{TR}}(\boldsymbol{r}, t)\right] \propto x(-t) *_{t} \mathcal{F}^{-1}\left\{\tilde{\boldsymbol{\rho}}\left(\boldsymbol{r}, \boldsymbol{r}^{\prime}, \omega\right) *_{\boldsymbol{r}^{\prime}} \overline{\boldsymbol{F}}\left(\hat{\boldsymbol{r}}^{\prime}, \omega\right)\right\},
$$

where $*_{\boldsymbol{r}^{\prime}}$ is the pseudo-convolution integral required in (16) and carried out over $\Sigma$. Comparing (30) with (29) it appears that in order to have a converging version of the target wavefront, $\tilde{\boldsymbol{\rho}}\left(\boldsymbol{r}, \boldsymbol{r}^{\prime}, \omega\right)$ should comply with the three following points: 1) it should provide a delay going like $r / c_{0} ; 2$ ) a radial dependence like $1 / r$ in the far-field region and 3) should not distort the angular dependence $\boldsymbol{F}(\hat{\boldsymbol{r}}, \omega)$. The first two points are easily verified since the functions (21) appearing in the PSF (26) are dominated by terms going like $\exp \left( \pm \mathrm{j} k_{0} r\right) / r$. As shown in the examples in section VIII, the coexistence within the PSF of the incoming and outgoing versions of the free-space propagator has a simple physical meaning: if a focusing wavefront is generated, after focusing onto the phase-center of the synthetic source, it will inevitably diverge along the opposite direction. The only point requiring a closer investigation is the third one, i.e., the eventual distortion of the angular dependence, which is not easily assessable from (16). 
To this effect, it is convenient to study the average wavefront $\mathrm{E}\left[\boldsymbol{E}_{\mathrm{TR}}(\boldsymbol{r}, \omega)\right]$ over the equivalent-source surface $\Sigma$. The fact that the PSF $\tilde{\boldsymbol{\rho}}\left(\boldsymbol{r}, \boldsymbol{r}^{\prime}, \omega\right)$ is dependent on the distance $r_{\Sigma} / \lambda$ is not due to limitations in the equivalent-source approach, but rather because as $r_{\Sigma}$ becomes smaller, while the wavefront in the far-field region shall not be modified, its progression towards the focal spot will indeed lead to a modification of the wavefront features, due to diffraction phenomena in the focal region [29].

Therefore, the properties of the PSF $\tilde{\boldsymbol{\rho}}\left(\boldsymbol{r}, \boldsymbol{r}^{\prime}, \omega\right)$ can be evaluated in a general manner by setting the dominant parameter $r_{\Sigma} / \lambda$, i.e., deciding how close the observer will be to the focal spot. The choice of having the equivalentsource surface coinciding with that of the observer allows a simpler comparison of how the radiation pattern (considered independent of the distance) will eventually be affected by the PSF. An example is given in Fig. 6, where the nine components of the PSF $\tilde{\boldsymbol{\rho}}\left(\boldsymbol{r}, \boldsymbol{r}^{\prime}, \omega\right)$ are shown for the case of vectors $\boldsymbol{r}$ and $\boldsymbol{r}^{\prime}$ belonging to the same surface $\Sigma$, where the choice $r_{\Sigma}=3 \lambda$ was made. The PSF is expressed with respect to a spherical reference system, for a point-source positioned at an $\boldsymbol{r}^{\prime}$ chosen to be along $\hat{\boldsymbol{x}}$.

Fig. 6 shows that the PSF is not perfectly isotropic, with diagonal terms depending on the field component, and offdiagonal terms (cross-polarization coupling) not identically equal to zero. The weak oscillations outside the peak regions present a zero mean-value and can be expected to lead to a very low result after the pseudo-convolution (16), as their overall contribution to the integral will be negligible as long as the target pattern undergoes angular variations slower than the pseudo-period of these oscillations. This point will be given more room in the rest of this section and in the next one.

The six off-diagonal terms share the absence of an evensymmetry positive-valued dominant peak, substituted by oddsymmetry responses. The low-level oscillations present patterns similar to those found in the three direct terms. Of particular interest is the fact that the coupling terms are stronger between the two tangential components $\vartheta$ and $\varphi$, which could be a source of inaccuracy in the reproduction of the target wavefront. Still, the $\hat{\boldsymbol{\vartheta}} \hat{\varphi}$ and $\hat{\varphi} \hat{\vartheta}$ terms are characterized by a double odd-symmetry, implying that convolution with a evensymmetric radiation pattern would result in a zero at $\boldsymbol{r}^{\prime}$, i.e., no coupling between the $\vartheta$ and $\varphi$ components. In other words, the original polarization should be expected to be preserved. Examples are shown in section VIII for the radiation pattern generated by an aperture source, where it is made clear how the coupling is indeed very weak and negligible in practical configurations. These results are consistent with those presented in [37], [38], extending them to the general case of a wavefront rather than a mere focal spot.

For the PSF $\tilde{\boldsymbol{\rho}}\left(\boldsymbol{r}, \boldsymbol{r}^{\prime}, \omega\right)$ to be expected not to modify the original radiation pattern, it should be real-valued: in any other case, it would at least imply a phase distortion of the wavefront. In practice the imaginary part of the PSF is much weaker than the real part, but for two components: $\hat{\boldsymbol{r}} \hat{\boldsymbol{\vartheta}}$ and $\hat{\boldsymbol{r}} \hat{\boldsymbol{\varphi}}$. As it will be shown in the examples presented in section VIII, the role of these two functions is to reproduce the natural distortion of the wavefront passing from essentially spherical (in the far-field region) to the one corresponding to the nearfield region of the synthetic source. This remark does not involve the idea of reproducing the reactive components of the field, as the focal region of the wavefront is usually found in the far-field region of the TRM antennas, the actual sources of the converging wavefront [29]. These considerations should be clearer when compared with the numerical results presented in section VIII.

The impact of the PSF on the angular dependence of $\boldsymbol{E}_{\mathrm{TR}}(\boldsymbol{r}, \omega)$ can be assessed by studying the zone of maximum correlation in the diagonal terms of the PSF. The null-tonull width of this main lobe can be computed from (21) and (24) and intercepts an angular resolution angle $\psi_{\rho}$ that can be expressed (in radians) in a general way as

$$
\psi_{\rho}=\alpha_{\rho} \frac{\lambda}{r_{\Sigma}},
$$

where $\alpha_{\rho}$ can take the values 0.89 or 1.45 depending on the direction along which the angle $\psi_{\rho}$ is measured, since the examples in Fig. 6 show that the PSF does not present a cylindrical symmetry. It is common practice in optics to approximate the size of the PSF to about a wavelength for the diffraction of waves propagating in free-space: we will adopt this approximation by taking $\alpha_{\rho} \simeq 1$, for the sake of simplicity. We can expect the TREC to be capable of reliably reproducing the target wavefront as long as the angular rate of variation of the radiation pattern of the wavefront is not faster than that allowed by its PSFs.

With reference to Fig. 2, the assumption of an observation surface $\Sigma$ in the far-field region of the synthetic source requires

$$
\frac{r_{\Sigma}}{\lambda}>8\left(\frac{r_{\Xi}}{\lambda}\right)^{2},
$$

implying that given the radius $r_{\Sigma}$ of the equivalent-source surface, the maximum dimensions of the volume $V_{\Xi}$ containing the synthetic source is given an upper bound, as well as its maximum gain [25]. A simple estimate can be derived by recalling that for a directive source, e.g., an aperture of maximum width $2 r_{\Xi}$, the $-3 \mathrm{~dB}$ angle $\psi_{F}$ of its main lobe can be approximated by

$$
\psi_{F} \simeq \frac{\lambda}{2 r_{\Xi}} .
$$

From (32), the maximum directivity of the synthetic source, or the minimum width of its main lobe, is

$$
\psi_{F}>\sqrt{\frac{2 \lambda}{r_{\Sigma}}} .
$$

We can now consider that the PSF will lead to a minor modification of the radiation pattern as long as $\psi_{\rho}$ is small enough with respect to the angular variation in $\boldsymbol{F}(\hat{\boldsymbol{r}}, \omega)$, as measured by its main lobe $\psi_{F}$. To this end, we introduce the quantity $R$

$$
R=\frac{\psi_{F}}{\psi_{\rho}},
$$

representing the angular resolution power of the TREC. A numerical analysis is proposed in section VIII, where the quality of the angular dependence of the wavefront produced by the TREC is assessed as a function of $R$. 


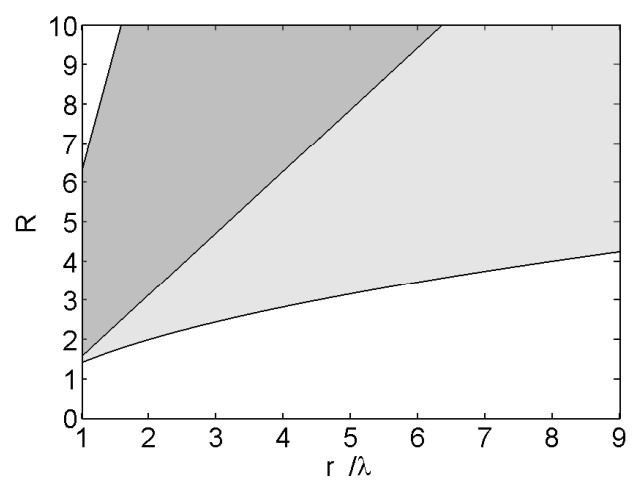

Fig. 7: Ranges of values taken by the ratio $R=\psi_{F} / \psi_{\rho}$, indicating the resolution power of the TREC. This quantity is plotted against the radius of $r_{\Sigma}$ of the equivalent-source surface for two cases: (a) the lightly shaded area corresponds to a cut along the $\mathrm{E}$ plane of the synthetic source, i.e., for a main lobe that is physically lower-bounded by that of a Hertzian dipole; (b) the domain of existence of the resolution power is extended to the more deeply-shaded area when dealing with the $\mathrm{H}$ plane of a linearly polarized source, i.e., its azimuthal plane, where its main lobe can present any value up to $2 \pi$ radian.

The range of values taken by $R$ can be estimated by recalling that $\psi_{F}$ is bounded as

$$
\psi_{\max }>\psi_{F}>\sqrt{\frac{2 \lambda}{r_{\Sigma}}} .
$$

The lower bound is actually due to the far-field assumption at the base of the proposed analysis, leading to (34), whereas the upper-bound comes from the fact that for a linearly polarized source the main-lobe $-3 \mathrm{~dB}$ angle is actually limited by a finite value. Considering the $\mathrm{E}$ plane of the synthetic source, the upper limit is given by $\psi_{\max }=\pi / 2$ radians for an Hertzian dipole, whereas for the $\mathrm{H}$ plane it can reach up $\psi_{\max }=2 \pi$ radians. Accounting for these bounds, the range of variation of $R$ is shown in Fig. 7, as a function of the electrical size of the equivalent-source surface $\Sigma$. It appears that $R>1.5$ as long as $\Sigma$ is at least one wavelength in radius. This lower limit is actually meaningful and even conservative, since any smaller choice would not allow the observer to be in the farfield of even the simplest source: indeed, the basic condition $k_{0} r_{\Sigma} \gg 1$ for the far-field region must also apply, hence requiring $r_{\Sigma} / \lambda \gtrsim 1.58$.

The results in Fig. 7 prove that for any far-field configuration, $R$ will always be higher than 1.5 , typically closer to 2 , i.e., radiation patterns will be at least twice larger than the PSFs main lobes.

\section{NUMERICAL RESULTS}

Numerical results are here presented in order to support our conclusions about the ability of the TREC to reproduce arbitrary wavefronts within a wave-diffusive medium. These have been obtained by numerically solving the convolution integral in (16), as applied to a reference radiation pattern.

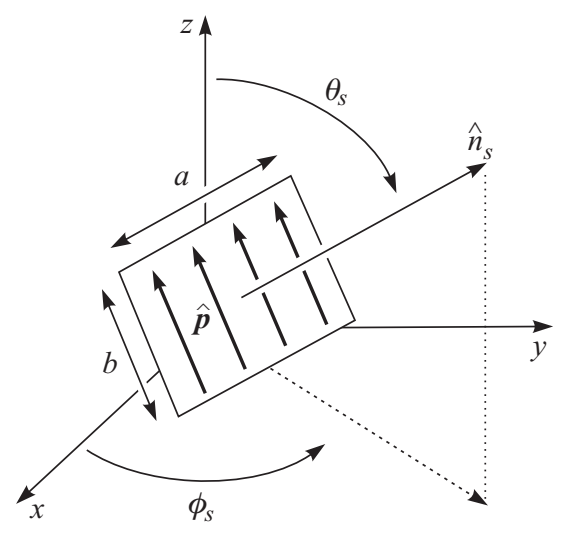

Fig. 8: The synthetic source considered in the validation presented in section VIII. It consists of an ideal rectangular aperture of dimensions $a$ and $b$, with a uniform field distribution linearly polarized along the direction $\hat{p}$.

Our choice was for a rectangular aperture, as the one shown in Fig. 8, with sides $a$ and $b$, characterized by a uniform electric field linearly polarized along the generic direction $\hat{p}$ lying on the aperture, radiating towards the half-space identified by $\hat{\boldsymbol{r}}$. $\hat{\boldsymbol{n}}_{s} \geqslant 0$.

Assuming an aperture initially lying on the $x y$ plane and its main-lobe radiating along $\hat{z}$, its radiation pattern can be approximated in closed-form as [26]

$$
\begin{aligned}
& \boldsymbol{F}_{\mathrm{wf}}(\hat{\boldsymbol{r}}, \omega)=(1+\cos \vartheta)(\hat{\boldsymbol{\vartheta}} \hat{\boldsymbol{\vartheta}}+\hat{\boldsymbol{\varphi}} \hat{\boldsymbol{\varphi}}) \cdot \hat{\boldsymbol{p}} \\
& \operatorname{sinc}\left(\pi \frac{a}{\lambda} \hat{\boldsymbol{x}} \cdot \hat{\boldsymbol{r}}\right) \operatorname{sinc}\left(\pi \frac{b}{\lambda} \hat{\boldsymbol{y}} \cdot \hat{\boldsymbol{r}}\right),
\end{aligned}
$$

while a generic orientation along the angles $\vartheta_{s}$ and $\varphi_{s}$ can be analyzed by applying standard rotation transformations.

We first proceed by assessing how accurately the radiation pattern is reproduced for a varying electrical distance from the synthetic-source volume, in the frequency domain, in section VIII-A. The existence of a focusing phenomenon is proven, while showing up to what distance the angular dependence dictated by target radiation pattern holds. We are particularly interested in assessing whether distortions of the wavefront intervene before its reaching the focal spot, which would imply a limitation in the accuracy of the TREC even during the far-field propagation of the wavefront. In a second time, section VIII-B elucidates the existence of a back-lobe contribution, by considering the time-domain evolution of the converging wavefront for a non-harmonic excitation.

\section{A. Frequency domain : focusing}

Thanks to (16) and (26), we computed the average field distribution that would be observed over concentric surfaces of radius $0.7,1,3$ and $5 \lambda$, for an aperture of sides $a=b=1 \lambda$ pointing towards $\vartheta_{s}=\pi / 2, \varphi_{s}=0$ with a $\hat{\boldsymbol{y}}$-polarized electric field (see Fig. 8). The results are shown in Fig. 9, together with the target radiation pattern, as a reference. The results are expanded into the three spherical coordinate components, with the target radiation pattern having no radial component (farfield radiation). It appears that the TREC approach is indeed 

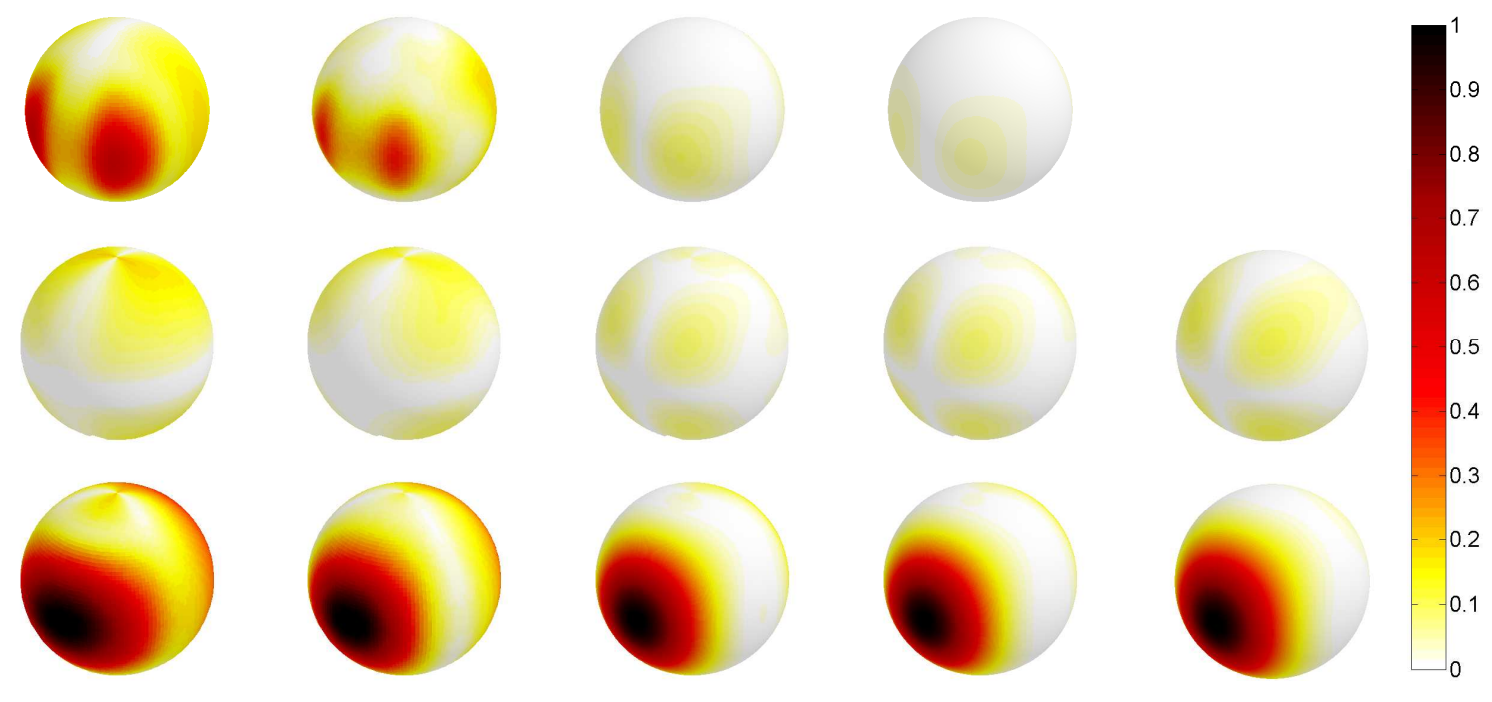

$r / \lambda=0.7$

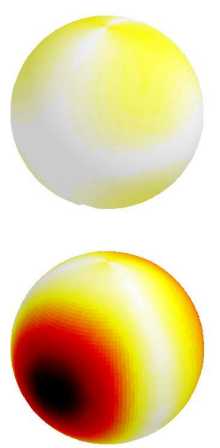

$r / \lambda=1$
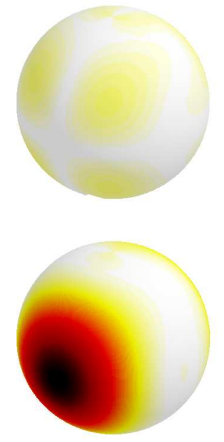

$r / \lambda=3$
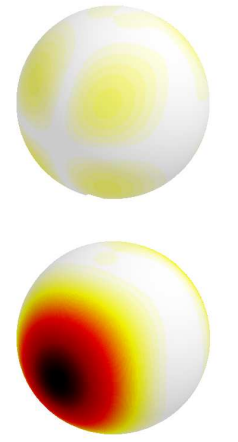

$r / \lambda=5$

Target wavefront

Fig. 9: The electric field distribution of the wavefront generated by a TREC as it would be observed at several distances. The side of incidence of the wavefront is shown, where the absolute value of the field is considered for the three spherical components of the field, in the order $E_{r}, E_{\vartheta}$ and $E_{\varphi}$ respectively, from the top to bottom. The target wavefront distribution is given as a reference in the last column. All results are normalized to the peak-amplitude of the $E_{\varphi}$ component, for each distance.

capable of very accurately reproducing the target radiation pattern even at very close distance, with non-negligible distortions occurring only within a one-wavelength distance from the phase-center of the synthetic source. The polarization is also preserved, not only the dominant component along $\hat{\varphi}$, but also the cross-polarization $\hat{\vartheta}$. A substantial deformation of the radiation pattern is observed at $0.7 \lambda$, where the PSF component for the $\hat{\varphi} \hat{\varphi}$ contribution is clearly recognizable, with a more elongated distribution along the $\hat{\varphi}$.

The back-lobe contribution appearing in Fig. 9 will be shown in section VIII-B to result from the impinging wave focusing through the phase-center of the synthetic-source and subsequently diverging along the opposite direction.

The same type of computation was carried out over a continuous range of distances from $\lambda / 10$ up to $3 \lambda$, limited to a horizontal cut, along the $x y$ plane, starting from an equivalentsource surface at $r_{\Sigma}=3 \lambda$. These results are shown in Fig. 10, where the two spherical components $E_{\varphi}(\boldsymbol{r}, \omega)$ and $E_{r}(\boldsymbol{r}, \omega)$ of the electric field are shown, $E_{\vartheta}(\boldsymbol{r})$ being identically null by virtue of symmetry. We therefore conclude that a focusing of the propagating energy is indeed occurring, as the electric field builds up converging towards the phase-center of the synthetic source.

Two notable distances are marked in Fig. 10: $r_{\Sigma}=2 \lambda$ and $r_{\Xi}=\lambda / 2$. The target wavefront (far field) should only present a $\hat{\varphi}$-oriented field, which is indeed found in the TRECgenerated wavefront, as shown in Fig. 10(a)-(b); the purity of the polarization appears to start degrading as the wavefront crossed $r_{\Xi}$, when the focusing wavefront approximate the original field distribution found in the reactive part of the synthetic source, i.e., $\hat{\boldsymbol{y}}$-oriented, as clearly visible in the vector representation in Fig. 11. Since the TREC, as any other anechoic environment, can only produce propagative waves by means of distant sources (i.e., the TRM antennas), the diffraction limit ensues, leading to a focal spot about one wavelength wide. The appearance of a radial component in Fig. 10(b) is due to this phenomenon of approximation of the original source distribution, and it becomes more evident when looking at the total field in Fig. 10(c): the wavefront focuses back onto the source region, with an almost uniform intensity.

The accuracy of the angular distribution of the focusing wavefront is more easily observed in Fig. 10(d), where the wavefront is normalized to Green's scalar function, yielding the radiation pattern to be compared to that shown in Fig. 10(e). The comparison is very good, with the converging wavefront accurately reproducing a constant radiation pattern over its far-field region within a $\pm 0.2 \mathrm{~dB}$ range over the main lobe. Fig. 10(d) also provides a clear picture of the focal spot due to diffraction limit: directivity is lost, with energy almost equally spread over all directions, and particularly with a reduction in its increase with respect to an ideal spherical convergence.

These results imply that the loss of directivity is not due to an intrinsic limitation of the method, as could have been expected from the PSF shown in the previous section. It actually appears that the PSF is effectively capable of reproducing all the phenomena leading to wave focusing under physical conditions, including the diffraction limitation over the nearfield region of the synthetic source. Practically, no significant distortion occurs over the main-lobe outside the surface $\Xi$, thus implying that there is no need to require $R \gg 1$ to avoid distortions of the far-field distribution of the wavefront.

The generated wavefront deviates from the target one outside the main lobe, even in the far-field region, within a range 

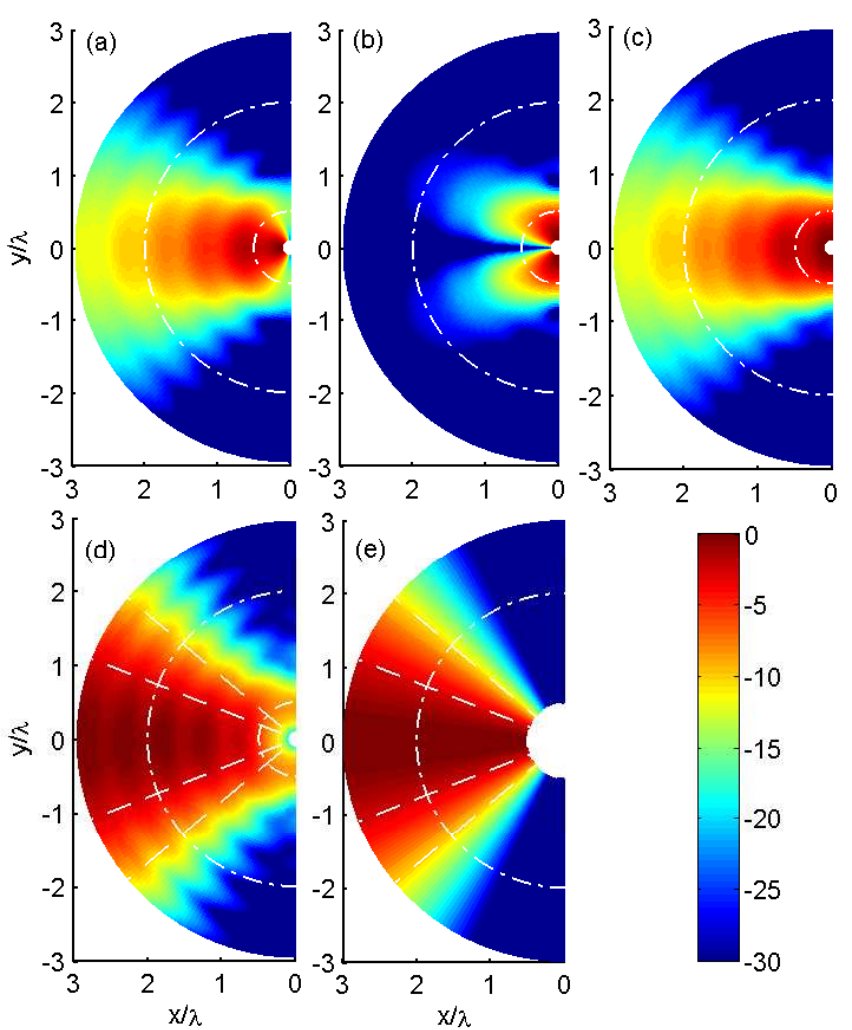

Fig. 10: Numerical solution of (16) for the case of the radiation pattern of the wideband aperture antenna described in the body of the text and shown in Fig. 8. The evolution of the electric field is studied over the half-plane of the $x y$ cut along which the time-reversed wavefront is expected to focus, for radial distances going from $\lambda / 10$ up to $3 \lambda$ : (a) $E_{\varphi}(\boldsymbol{r})$; (b) $E_{r}(\boldsymbol{r})$; (c) $\|\boldsymbol{E}(\boldsymbol{r})\|$; (d) $\|\boldsymbol{E}(\boldsymbol{r}) / G(r)\|$; (e) angular dependence $\|\boldsymbol{F}(\boldsymbol{r})\|$, proportional to $\left\|\boldsymbol{E}_{\mathrm{wf}}(\boldsymbol{r}) / G(r)\right\|$. The outer dashed line represents the Fraunhofer distance for the synthetic source, whose volume is marked by the inner dashed line. All results are normalized to the peak-value of $E_{\varphi}$ and expressed in dB. Radial dashed lines represent the $-3 \mathrm{~dB}$ and the $-10 \mathrm{~dB}$ angles.

of values inversely related to the intensity of the field. The reason for this phenomenon is not clear and deserves further investigations. It could indeed be caused by the approximate radiation pattern (37) used as target, or to intrinsical limitations in the TREC.

\section{B. Time domain : causality and back-lobe radiation}

The results in Fig. 9 present a back-lobe radiation related to the long-range correlation of the PSF. Its physical meaning becomes clear when studying the PSF in the time domain. We have considered the same case as in the previous section, with an equivalent-source surface of radius $r_{\Sigma}=3 \lambda$, imposing an unchanged radiation pattern over a relative bandwidth $B_{T} / f_{c}=10 \%$ around the central frequency $f_{c}$ of the wavefront excitation. All results are normalized to the central frequency.

Following these choices, the field distribution over the $x y$ cut of $\Sigma$ was computed in the time domain, yielding the results
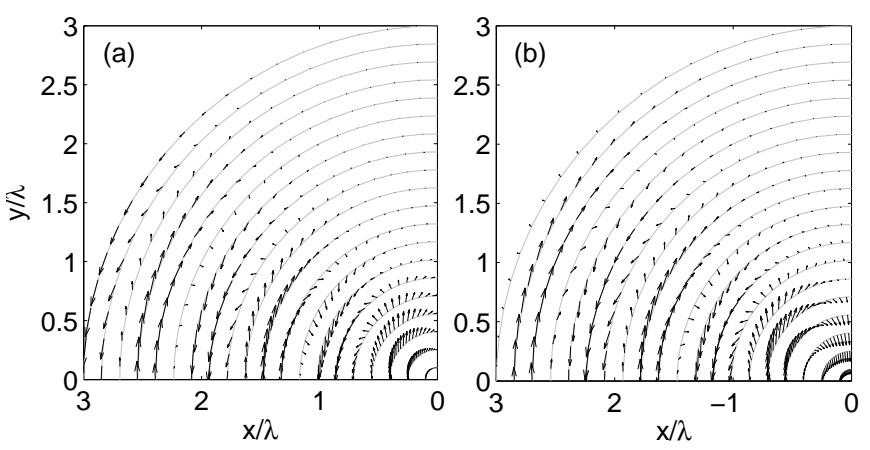

Fig. 11: Vector representation of the normalized electric field $\mathrm{E}\left[\boldsymbol{E}_{\mathrm{TR}}(\boldsymbol{r}, \omega)\right] / G(r, \omega)$ shown in Fig. 10: (a) real and (b) imaginary part. As the wavefront closes onto the focal region, the electric field passes from the TEM configuration typical of far-field radiation to a $\hat{\boldsymbol{y}}$-oriented configuration, reminiscent of the original field distribution of the synthetic aperture.

shown in Fig. 12. Here, the field over the $x y$-cut of $\Sigma$ is shown as an angular distribution function of the time, proving that the back lobe is actually the time-delayed replica of the impinging wavefront. The delay $\tau$ is equal to a normalized delay $f_{c} \tau=6$, which coincides with a free-space propagation across a sphere of radius $3 \lambda$, since $f_{c} \tau=2 r_{\Sigma} / \lambda=6$. Causality is thus preserved, indicating that the proposed model is capable of correctly assessing time-domain phenomena. In particular, the previous results dealing with the focusing of energy can be rightly interpreted as due to a convergent wavefront, while the presence of the back-lobe is necessary for the causality of the solution yielded by (16).

A non-ideality of the TREC method is apparent in Fig. 12(b): the wavefront emerging from the focal region is slightly distorted for the directions away from the main lobe. This fact corresponds to the an error in the position of the focusing spot of about $\lambda / 8$, a fact that leaves room to the interpretation of these errors as due to the use of the approximate model (37). This notwithstanding, these errors do not affect the conclusions of our work, as the proposed method is clearly capable of a remarkably accurate reproduction of free-space propagation within a wave-diffusive medium.

\section{Summary AND DISCUSSIONS}

We reckon that at this point it is important to summarize the main results and ideas introduced in this paper. The concept of time-reversed wavefronts has been revisited in a novel manner, by looking at an originally diverging wavefront as being radiated by equivalent currents, as opposed to the standard approach based on a physical (and often pointlike) source. This different approach allows the introduction of synthetic sources, which can be characterized by ideal features not easily found in real-life sources. Moreover, these features can be changed in real-time through a simple postprocessing procedure, yielding new excitation signals to be applied to the ports of the TRM antennas. As a result, a wavediffusive medium can be "converted" into an anechoic one where deterministic wavefronts propagate as in a free-space 


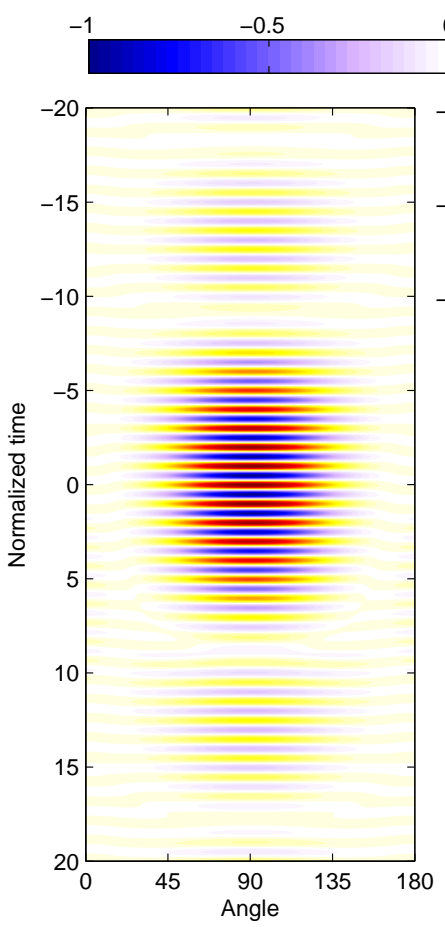
0 0.5

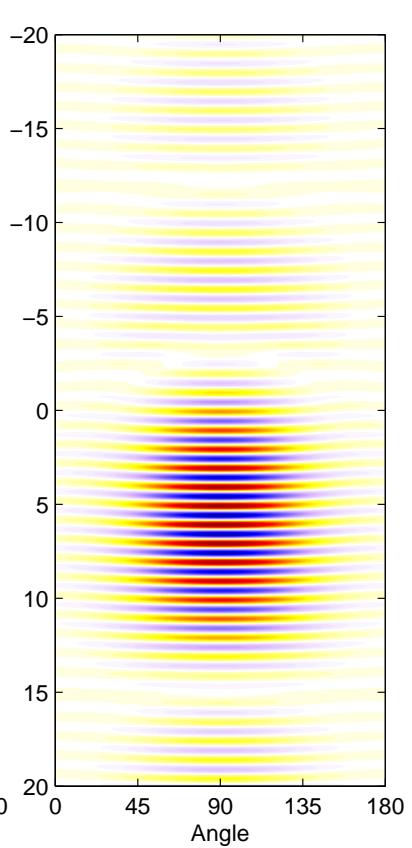

Fig. 12: Time-domain results for the $\hat{\varphi}$ component of the average field generated by a TREC, as computed over a spherical surface of radius $r_{\Sigma}=3 \lambda$, for a relative bandwidth $B_{T} / f_{c}=10 \%$. The signal was chosen to have a constant spectrum over $B_{T}$, i.e., to have a sine cardinal profile in the time domain. The results refer to the $x y$-cut of : (a) the converging wavefront impinging over $\Sigma$, along the negative part of the $x$-axis; (b) the diverging wavefront observed on the opposed direction.

environment; interestingly, no hypothesis is needed about an eventual link between the direction of arrival of the wavefront and the positions of the TRM antennas, thanks to the diffusive nature of the medium.

This surprising result entirely relies on the knowledge of Green's functions between the equivalent-source surface $\Sigma$ and the TRM antennas. These data can be readily measured by means of low-scattering probes moved over $\Sigma$, as describe in [8]. Previous experimental validations of the TREC approach are indeed already available in the literature. In this paper we rather aimed at providing a deeper insight into the physics of the TREC, from a theoretical point of view, in order to have a better understanding of its intrinsical limitations, whence our emphasizing theoretical and numerical results.

What is most surprising is that the coupling of time-reversed excitations to a diffusive cavity through the use of synthetic sources allows generating any kind of wavefront, on average. The question of how far the generated wavefront is from the average one can be directly assessed by recalling the concept of intrinsical SNR due to the inevitable finite number of degrees of freedom available within the cavity. This issue was studied in [11] and the main results are recalled in Appendix B, where it is shown that the actual response of a TREC is very close to its ensemble average.
The potential use of the TREC as a new kind of testing facility clearly implies the inclusion of an EUT into the test-volume defined by $\Sigma$. It goes without saying that the presence of the EUT can have a dramatic impact on the Green's functions that would be observed with and without the EUT. As a result, the EUT needs to be present during the characterization phase yielding the $\boldsymbol{N}_{\mathrm{eq}, i}\left(\boldsymbol{r}, \boldsymbol{r}^{\prime}, \omega\right)$ functions. Without entering into details, the presence of an EUT could impact the resulting wavefront at two levels: 1) by modifying the wavefront radiated by the equivalent currents; 2) by modifying the Green's functions within the cavity. The first point is actually not a real issue, since the equivalence theorem ensures that the use of electric and magnetic currents implies that only an outwards radiation (first phase) would take place, thus not interacting with the EUT found within $\Sigma$. As a result, the average wavefront generated by the TREC should be expected to be the same with and without an EUT, as long as the diffusefield assumption holds, thanks to the self-averaging property of time reversal [27]. This point is fundamental if the TREC is to be used as a testing facility, since a reproducible wavefront independent of the EUT position, orientation and nature is a necessary condition for any metrology application.

A crucial issue is the question of the energy efficiency of the proposed procedure. We have not considered this point in the context of this paper, but it had already received attention in a previous work [39], where it was shown that the TREC also improves the ability to generate high-intensity peaks of electromagnetic power by a factor easily exceeding one hundred, with respect to standard harmonic excitations in an RC.

From a practical point of view, the simplifying assumptions used throughout the paper should be taken for what they are, i.e., not requirements, but just simplifications. For example, whenever the equivalent-current surface is not in the farfield region of the synthetic source, the TREC will reproduce the propagative part of the radiation pattern, filtering out the reactive part; the often dispersive response of electronic devices and antennas can be compensated when synthesizing the excitation signals; the average coupling between the TRM antennas can be kept as low as needed since they are operated in a wave-diffusive medium, i.e., with a weak spatial correlation.

\section{Conclusions}

In this paper we have investigated the potential advantages of applying TR techniques to tests based on the use of predefined wavefronts. Having found that these are not straightforwardly suitable for this purpose, an alternative approach, the TREC, has been introduced. A theoretical analysis has proved how the TREC enables a number of once held impossible features in a reverberation chamber, namely the generation of short-pulsed fields and a detailed control of the parameters of wavefronts. More specifically, the use of TR leads to an equivalent Green's function that appears to be very close to that of a free-space environment. The TREC is hence capable of recreating within a reverberation chamber the necessary conditions for the arbitrary generation of wavefronts. 
As a result, it was shown that the TREC allows the definition of a testing environment sharing the advantages of anechoic and reverberation chambers, namely the possibility of knowing exactly the type of EM wavefront (polarization, direction of arrival, time-dependence) testing the EUT response, while maintaining the high energetic efficiency of reverberation chambers. Furthermore, it was shown that the test wavefront can be ideally steered in real-time, without requiring any mechanical displacement of the antennas. The use of faststeered deterministic test wavefronts could lead to faster R\&D cycles, giving a clearer information about the response of an EUT to impinging wavefronts.

\section{APPENDIX A}

RMS VALUE OF THE $\boldsymbol{N}(\boldsymbol{r}, \omega)$ TRANSFER FUNCTIONS FOR A DIFFUSIVE REVERBERATION CHAMBER

Following the conventions introduced in section VI, the field radiated by a TRM antenna is given by

$$
\boldsymbol{E}(\boldsymbol{r}, \omega)=N_{\mathrm{e}}(\boldsymbol{r}, \omega) \frac{V_{\mathrm{ex}}(\omega)}{Z_{\mathrm{ant}}(\omega)} h_{e}(\omega) .
$$

Assuming a purely resistive input impedance for the antennas and a perfectly matched generator leads to

$$
\frac{\|\boldsymbol{E}(\boldsymbol{r}, \omega)\|^{2}}{P_{\mathrm{av}}(\omega)}=\frac{4\left|h_{e}(\omega)\right|^{2}}{Z_{\mathrm{ant}}(\omega)}\left\|\boldsymbol{N}_{\mathrm{e}}(\boldsymbol{r}, \omega)\right\|^{2},
$$

with $P_{\mathrm{av}}(\omega)$ the available power of the generator. Computing the ensemble average of (39) and recalling the uniformity property for the electric field in a diffusive cavity yields

$$
\begin{aligned}
N_{\mathrm{e}, \mathrm{av}}^{2}(\omega) & =\mathrm{E}\left[\left\|\boldsymbol{N}_{\mathrm{e}}(\boldsymbol{r}, \omega)\right\|^{2}\right] \\
& =\frac{Z_{\mathrm{ant}}(\omega)}{4\left|h_{e}(\omega)\right|^{2}} \mathrm{E}\left[\frac{\|\boldsymbol{E}(\boldsymbol{r}, \omega)\|^{2}}{P_{\mathrm{av}}(\omega)}\right],
\end{aligned}
$$

thus showing how $N_{\mathrm{e}, \mathrm{av}}^{2}(\omega)$ is related to the average energy efficiency of the cavity $\mathrm{E}\left[\|\boldsymbol{E}(\boldsymbol{r}, \omega)\|^{2} / P_{\mathrm{av}}(\omega)\right]$ through the electrical parameters of the TRM antenna. For the special case of a reverberation chamber, the energy efficiency can be estimated in a straightforward manner as [35]

$$
\mathrm{E}\left[\frac{\|\boldsymbol{E}(\boldsymbol{r}, \omega)\|^{2}}{P_{\mathrm{av}}(\omega)}\right]=\frac{4 \zeta_{0}}{\pi} \frac{Q(\omega) \lambda}{V} .
$$

where $Q(\omega)$ is the average composite quality factor of the cavity, $V$ is the volume of the medium filling it and $\lambda$ the average wavelength corresponding to the frequency of excitation of the cavity. This result is straightforwardly linked to the variance of any Cartesian component of the electric field and the covariance of orthogonal components of the electric and magnetic field as

$$
\begin{aligned}
\mathrm{E}\left[\left|\hat{\boldsymbol{u}}_{m} \cdot \boldsymbol{N}_{\mathrm{e}}(\boldsymbol{r}, \omega)\right|^{2}\right] & =\frac{N_{\mathrm{e}, \mathrm{av}}^{2}(\omega)}{3}(42 \mathrm{a}) \\
\mathrm{E}\left[\hat{\boldsymbol{u}}_{m} \cdot \boldsymbol{N}_{\mathrm{e}}(\boldsymbol{r}, \omega) \hat{\boldsymbol{u}}_{n} \cdot \overline{\boldsymbol{N}}_{\mathrm{m}}(\boldsymbol{r}, \omega)\right] & =\frac{N_{\mathrm{e}, \mathrm{av}}^{2}(\omega)}{3 \zeta_{0}}(42 \mathrm{~b})
\end{aligned}
$$

with $\hat{\boldsymbol{u}}_{m} \cdot \hat{\boldsymbol{u}}_{n}=0$. The above result relies on the average isotropy of the electric and magnetic fields in a diffusive cavity.

\section{APPENDIX B}

\section{FLUCTUATIONS IN TIME-REVERSED WAVEFRONTS}

The reason why the results presented in this paper always deal with ensemble averages is the self-averaging property typical of non-harmonic TR applications [27], [28]. This property is inherited by the fact that for a finite bandwidth $B_{T}$ of excitation, the coherent excitation of a complex medium implies an average over $B_{T}$, that can be shown to approximate an ensemble average.

For the special case of bounded media with low losses, it has been shown that time-reversed signals are affected by a residual error in the transmission through the medium, due to the physical impossibility of efficiently transmitting certain spectral components in a steady-state configuration. As soon as frequency-selective media are considered, this residual error, intrinsical to the very procedure of time-reversal transmissions, can be assimilated to a background noise, or intrinsical noise. Its rms intensity can be straightforwardly linked to a few parameters, such as the average composite quality factor $Q\left(f_{c}\right)$ evaluated at the central frequency and the fractional bandwidth $B_{T} / f_{c}$ [11]. By defining the intrinsical SNR $\Lambda_{p}$ as the ratio between the peak instantaneous power of the coherent part of the time-reversed signal and the rms power of the residual noise, it can be proven that

$$
\Lambda_{p}=\Lambda \frac{Q\left(f_{c}\right) \kappa^{2}}{\pi} \frac{B_{T}}{f_{c}},
$$

where $\Lambda$ is the energy SNR, which can be shown to be close to one for a diffusive medium [11], while $\kappa$ is the ratio of the real part of the average of $X(\omega)$ over its rms value, both defined over the bandwidth $B_{T}$.

Assuming the residual error to behave as a normallydistributed random variable, its rms amplitude coincides with its standard deviation $\sigma_{n}$. The confidence interval within which the fluctuations are expected to be found with a probability of, e.g., $95 \%$, is thus simply given by the interval $\pm 2 \sigma_{n}$, resulting in a relative confidence margin around the peak value of the transmitted signal

$$
\frac{\Delta E}{E}=\frac{2}{\sqrt{\Lambda_{p}}}=\frac{2}{\kappa} \sqrt{\frac{\pi f_{c}}{Q\left(f_{c}\right) B_{T}}} .
$$

As a practical example, let us consider a moderately resonant cavity with $Q=5 \cdot 10^{4}, B_{T} / f_{c}=5 \%$ and a sine cardinal signal excitation, i.e., $\kappa=1$, yielding a range of fluctuations of about $\pm 7 \%$, with a confidence of $95 \%$. Such a low level of deviations is the reason why having access to a model of the average response is indeed representative of the actual response observed for a specific configuration.

\section{REFERENCES}

[1] J. Toivanen, T. Laitinen, V. Kolmonen, and P. Vainikainen, "Reproduction of arbitrary multipath environments in laboratory conditions," Instrumentation and Measurement, IEEE Transactions on, vol. 60, no. 1, pp. $275-281$, jan. 2011.

[2] D. Ward and T. Abhayapala, "Reproduction of a plane-wave sound field using an array of loudspeakers," Speech and Audio Processing, IEEE Transactions on, vol. 9, no. 6, pp. 697 -707, sep 2001. 
[3] P. Kildal, "Overview of 6 years R\&D on characterizing wireless devices in Rayleigh fading using reverberation chambers," in International Workshop on Antenna Technology: Small and Smart Antennas Metamaterials and Applications, 2007. IWAT'07. IEEE, 2007, pp. 162-165.

[4] D. Hill, "Plane wave integral representation for fields in reverberation chambers," IEEE Transactions on Electromagnetic Compatibility, vol. 40, no. 3, pp. 209-217, 1998.

[5] P. Corona, G. Ferrara, and M. Migliaccio, "Reverberating chambers as sources of stochastic electromagnetic fields," IEEE Transactions on Electromagnetic Compatibility, vol. 38, no. 3, pp. 348 -356, Aug 1996.

[6] P.-S. K. M. Bäckström, O. Lundén, "Reverberation chambers for emc susceptibility and emission analyses," in Review of Radio Science 19992002, 2002, pp. 429-452.

[7] J. de Rosny, G. Lerosey, and M. Fink, "Theory of electromagnetic timereversal mirrors," IEEE Transactions on Antennas and Propagation, vol. 58, no. 10, pp. 3139-3149, 2010.

[8] H. Moussa, A. Cozza, and M. Cauterman, "Experimental demonstration of directive pulsed wavefront generation in reverberation chambers," Electronics Letters, vol. 46, no. 9, pp. 623-624, 2010.

[9] C. Oestges, A. Kim, G. Papanicolaou, and A. Paulraj, "Characterization of space-time focusing in time-reversed random fields," IEEE Transactions on Antennas and Propagation, vol. 53, no. 1, pp. 283 - 293, jan. 2005.

[10] H. Moussa, A. Cozza, and M. Cauterman, "Directive wavefronts inside a time reversal electromagnetic chamber," in IEEE EMC Symposium, Austin, Texas, 2009., 2009.

[11] A. Cozza, "Statistics of the performance of time reversal in a lossy reverberating medium," Physical Review E, vol. 80, no. 5, p. 56604, 2009.

[12] A. Yariv, "Phase conjugate optics and real-time holography," IEEE Journal of Quantum Electronics, vol. 14, no. 9, pp. 650-660, 1978.

[13] D. Cassereau and M. Fink, "Time-reversal of ultrasonic fields. III. Theory of the closed time-reversal cavity," IEEE Transactions on Ultrasonics, Ferroelectrics and Frequency Control, vol. 39, no. 5, pp. 579592, 2002.

[14] C. Draeger and M. Fink, "One-channel time-reversal in chaotic cavities: Theoretical limits," The Journal of the Acoustical Society of America, vol. 105 , p. $611,1999$.

[15] G. Lerosey, J. de Rosny, A. Tourin, A. Derode, G. Montaldo, and M. Fink, "Time reversal of electromagnetic waves," Physical Review Letters, vol. 92, no. 19, p. 193904, 2004.

[16] P. Roux, B. Roman, and M. Fink, "Time-reversal in an ultrasonic waveguide," Applied Physics Letters, vol. 70, p. 1811, 1997.

[17] E. Joy and D. Paris, "Spatial sampling and filtering in near-field measurements," IEEE Transactions on Antennas and Propagation, vol. 20, no. 3, pp. 253-261, 1972.

[18] Environmental Conditions and Test Procedures for Airborne Equipment, Radio Technical Commission for Aeronautics (RTCA) Std. DO-160E, 2005.

[19] C. Prada and M. Fink, "Eigenmodes of the time reversal operator: A solution to selective focusing in multiple-target media," Wave Motion, vol. 20, no. 2, pp. 151-163, 1994.

[20] F. Gruber, E. Marengo, and A. Devaney, "Time-reversal imaging with multiple signal classification considering multiple scattering between the targets," The Journal of the Acoustical Society of America, vol. 115, p. 3042, 2004.

[21] W. Kuperman, W. Hodgkiss, H. Song, T. Akal, C. Ferla, and D. Jackson, "Phase conjugation in the ocean: Experimental demonstration of an acoustic time-reversal mirror," The Journal of the Acoustical Society of America, vol. 103, p. 25, 1998.

[22] G. Lerosey, J. De Rosny, A. Tourin, A. Derode, G. Montaldo, and M. Fink, "Time reversal of electromagnetic waves and telecommunication," Radio Science, vol. 40, no. 6, 2005.

[23] M. Yavuz and F. Teixeira, "Full time-domain dort for ultrawideband electromagnetic fields in dispersive, random inhomogeneous media," IEEE Transactions on Antennas and Propagation, vol. 54, no. 8, pp. 2305-2315, 2006

[24] R. Sullivan, Microwave radar: Imaging and advanced concepts. Artech House, Inc. Norwood, MA., 2000.

[25] R. Harrington, "On the gain and beamwidth of directional antennas," IRE Transactions on Antennas and Propagation, vol. 6, no. 3, pp. 219$225,1958$.

[26] — Time-Harmonic Electromagnetic Fields. McGraw-Hill, New York, NY, 1961.

[27] A. Derode, A. Tourin, and M. Fink, "Random multiple scattering of ultrasound. II. Is time reversal a self-averaging process?" Physical Review E, vol. 64, no. 3, p. 36606, 2001.
[28] J. Fouque and G. Papanicolaou, Wave Propagation and Time Reversal in Randomly Layered Media. Springer Verlag, 2007.

[29] J. Stamnes, Waves in focal regions: propagation, diffraction, and focusing of light, sound, and water waves. Taylor \& Francis, 1986.

[30] C. Draeger, J. Aime, and M. Fink, "One-channel time-reversal in chaotic cavities: Experimental results," The Journal of the Acoustical Society of America, vol. 105, p. 618, 1999.

[31] A. Ishimaru, Wave propagation and scattering in random media. WileyIEEE Press, 1999, vol. 12

[32] J. Davy, "The relative variance of the transmission function of a reverberation room," Journal of Sound and Vibration, vol. 77, no. 4, pp. $455-479,1981$.

[33] A. Cozza, "The Role of Losses in the Definition of the Overmoded Condition for Reverberation Chambers and Their Statistics," IEEE Transactions on Electromagnetic Compatibility, no. 53, pp. 296-307, 2010.

[34] D. Hill and J. Ladbury, "Spatial-correlation functions of fields and energy density in a reverberation chamber," IEEE Transactions on Electromagnetic Compatibility, vol. 44, no. 1, pp. 95-101, 2002.

[35] D. Hill, "Electromagnetic Theory of Reverberation Chambers," National Institute of Standards and Technology, Tech. Rep., 1998.

[36] E. Wolf, Introduction to the Theory of Coherence and Polarization of Light. Cambridge University Press, 2007.

[37] A. Cozza and H. Moussa, "Enforcing deterministic polarisation in a reverberating environment," Electronics Letters, vol. 45, no. 25, pp. 1299-1301, 2009.

[38] _ _ "Polarization selectivity for pulsed fields in a reverberation chamber," in 2010 Asia-Pacific Symposium on Electromagnetic Compatibility (APEMC). IEEE, 2010, pp. 574-577.

[39] A. Cozza, "Increasing peak-field generation efficiency of reverberation chamber," Electronics Letters, vol. 46, no. 1, pp. 38-39, 2010. 